\title{
Robust Adaptive Fuzzy Control for a Class of Uncertain MIMO Nonlinear Systems with Input Saturation
}

\author{
Shenglin Wen and Ye Yan \\ College of Aerospace Science and Engineering, National University of Defense Technology, Changsha 410073, China \\ Correspondence should be addressed to Shenglin Wen; wenshenglin1108@gmail.com
}

Received 11 November 2014; Revised 30 January 2015; Accepted 3 February 2015

Academic Editor: Yan-Jun Liu

Copyright ( 92015 S. Wen and Y. Yan. This is an open access article distributed under the Creative Commons Attribution License, which permits unrestricted use, distribution, and reproduction in any medium, provided the original work is properly cited.

\begin{abstract}
This paper studies the robust adaptive fuzzy control design problem for a class of uncertain multiple-input and multiple-output (MIMO) nonlinear systems in the presence of actuator amplitude and rate saturation. In the control scheme, fuzzy logic systems are used to approximate unknown nonlinear systems. To compensate the effect of input saturations, an auxiliary system is constructed and the actuator saturations then can be augmented into the controller. The modified tracking error is introduced and used in fuzzy parameter update laws. Furthermore, in order to deal with fuzzy approximation errors for unknown nonlinear systems and external disturbances, a robust compensation control is designed. It is proved that the closed-loop system obtains $H_{\infty}$ tracking performance through Lyapunov analysis. Steady and transient modified tracking errors are analyzed and the bound of modified tracking errors can be adjusted by tuning certain design parameters. The proposed control scheme is applicable to uncertain nonlinear systems not only with actuator amplitude saturation, but also with actuator amplitude and rate saturation. Detailed simulation results of a rigid body satellite attitude control system in the presence of parametric uncertainties, external disturbances, and control input constraints have been presented to illustrate the effectiveness of the proposed control scheme.
\end{abstract}

\section{Introduction}

In most practical control applications, such as those in robot manipulation and aerospace industry, the performance of the controller is directly related to the accuracy of the mathematical model and external disturbances. However, it is difficult to establish an appropriate mathematical model for a large number of nonlinear systems when the systems are complex and highly coupled nonlinear with structured uncertainties and external disturbances [1]. To tackle with this problem, fuzzy logic systems and neural networks have been extensively used in complex and ill-defined nonlinear systems due to their approximation ability of dealing with the nonlinear smooth functions [2]. Many adaptive fuzzy control and adaptive neural network control schemes have been developed for single-input and single-output (SISO) nonlinear systems [3-7], MIMO nonlinear systems [8-17], and SISO/MIMO nonlinear systems with immeasurable states [18-20], respectively. Generally, these adaptive fuzzy and neural network control approaches can achieve nice control performance without control saturation. If physical actuators saturation such as magnitude and rate constraints is considered, the adaptive intelligent control approaches mentioned above can not be implemented [21].

As we know, in many practical dynamic systems, physical actuators saturation on hardware indicates an inevitable constraint of the magnitude and rate limitations of the control signal. For example, due to physical limitation, momentum exchange devices or thrusters as actuator for the satellite attitude control system fail to render infinite control torque and thus the actuator can only provide limited control torques within a limited rate [22]. Control saturation is one of the most common nonsmooth nonlinearity that should be explicitly considered in the control design. The controllers that ignore actuator limitations may give rise to undesirable inaccuracy, severely degrade the performance of system, or even damage the stability of system [23]. Hence, the controller design subjected to the control saturation while simultaneously achieving higher performance is a very practical problem. 
The design of tracking controllers for uncertain MIMO nonlinear systems with actuator constraints is a challenging problem. During the past decades, there have been extensive researches on the control of nonlinear systems with various constraints. Analysis and design of control systems with control saturation have been widely studied in [24-42]. Farrell et al. [24-26] have presented an adaptive backstepping approach for unknown nonlinear systems with known magnitude, rate, and bandwidth constraints on intermediate states or actuators without disturbance. To tackle with the physical saturation, an auxiliary system with the same order as that of the plant was constructed to compensate the effect of saturation. The control input saturation is investigated through online approximation based control for uncertain nonlinear systems in [26]. An adaptive control and the constrained adaptive control in combination with the backstepping technique are proposed in [29]. A direct adaptive fuzzy control approach for uncertain nonlinear systems with input saturation is presented in [30], in which a Nussbaum function is used to compensate for the nonlinear term arising from the input saturation. In [31], an adaptive fuzzy output feedback control algorithm for a class of output constrained uncertain nonlinear systems with input saturation is developed by employing a barrier Lyapunov function and an auxiliary system. Adaptive backstepping tracking control based on fuzzy neural networks is investigated for unknown chaotic systems in [32] and with control input constraints in [33]. Neural network based adaptive control schemes with external disturbances and actuator saturations are presented in [35], in which auxiliary systems are added to attenuate the effects of input saturation. It is apparent that the presence of input saturation constraint substantially increases the complexity of control system design for uncertain MIMO nonlinear systems. In the constrained adaptive control, the key problem is how to handle the constraint effect of the actuator's physical constraints. To this end, we introduce an auxiliary design system to handle the constraint effect in this paper. Based on the states of the auxiliary design system, constrained adaptive control is investigated for a class of uncertain MIMO nonlinear systems with input constraints using robust fuzzy control technique.

In this paper, a robust adaptive fuzzy tracking control scheme is presented to handle the external disturbances and actuator physical constraints for uncertain MIMO nonlinear systems. In control design, fuzzy logic systems are used to approximate unknown nonlinear systems. Note that input saturations are nonsmooth functions but the adaptive fuzzy control technique requires all functions differentiable [6]. To compensate the effect of input saturations, an auxiliary system is constructed and the actuator saturations then can be augmented into the controller. The modified tracking error is introduced and used in fuzzy parameter update laws. Besides, in order to deal with fuzzy approximation errors for unknown nonlinear systems and external disturbances, a robust compensation control is designed. It is proved that the proposed control approach can guarantee that all the signals of the resulting closed-loop system are bounded, and the closed-loop system obtains $H_{\infty}$ tracking performance through Lyapunov analysis. The transient modified tracking errors performance is derived to be explicit functions of design parameters and thus bounds of modified tracking errors can be adjusted by tuning design parameters.

The rest of this paper is organized as follows. The description of the uncertain MIMO nonlinear system under consideration and necessary preliminaries are given in Section 2. In Section 3, the robust adaptive fuzzy control without control saturation is firstly designed. When the actuators have physical limitations, this approach may not be able to be successfully implemented. In order to solve this problem, a robust adaptive fuzzy control scheme with input saturation is investigated. The simulation results of satellite attitude control are presented to demonstrate the effectiveness of proposed controller in Section 4. Section 5 contains the conclusion.

\section{Problem Formulation and Preliminary}

2.1. Problem Formulation. Consider a class of uncertain MIMO affine nonlinear system

$$
\begin{gathered}
\dot{\mathbf{x}}=\mathbf{f}(\mathbf{x})+\sum_{i=1}^{m} \mathbf{g}_{i}(\mathbf{x}) u_{i}+\mathbf{d}^{\prime} \\
y_{1}=h_{1}(\mathbf{x}) \\
\vdots \\
y_{m}=h_{m}(\mathbf{x})
\end{gathered}
$$

where $\mathbf{x}=\left[x_{1}, \ldots, x_{n}\right] \in R^{n}$ is the state vector available for measurement; $\mathbf{u}=\left[u_{1}, \ldots, u_{m}\right] \in R^{m}$ is the control vector with $\left|u_{i}\right| \leq u_{i \max }$, where $u_{i \max }$ denote the actuator amplitude; $\mathbf{y}=\left[y_{1}, \ldots, y_{m}\right] \in R^{m}$ is the output vector; $h_{1}(\mathbf{x}), \ldots, h_{m}(\mathbf{x})$ are smooth functions defined on the open set of $\mathbf{R}^{n} . \mathbf{f}(\mathbf{x})$, $\mathbf{g}_{1}(\mathbf{x}), \ldots, \mathbf{g}_{m}(\mathbf{x})$ are continuous unknown but smooth vector fields. $\mathbf{d}^{\prime}=\left[d_{1}^{\prime}, \ldots, d_{n}^{\prime}\right]^{T} \in \mathbf{R}^{n}$ is external disturbance vector, where $d_{1}^{\prime}$ is unknown but bounded.

Define that $L_{\mathbf{f}} h_{i}$ is the Lie derivative of $h_{i}$ along vector field $\mathbf{f}(\mathbf{x})$ and $L_{\mathbf{f}}^{k} h_{i}$ is recursively as $L_{\mathbf{f}}^{k} h_{i}=L_{\mathbf{f}}\left(L_{\mathbf{f}}^{k-1} h_{i}\right)$. A multivariable uncertain nonlinear system of the form (1) has a vector relative degree $\left[r_{1}, r_{2}, \ldots, r_{m}\right]$ at a point $\mathbf{x}_{0}$ if

$$
L_{\mathbf{g}_{j}} L_{\mathbf{f}}^{k} h_{i}(\mathbf{x})=0 \quad \forall 1 \leq j \leq m, k \leq r_{i}-1
$$

for all $\mathbf{x}$ in a neighborhood of $\mathbf{x}_{0}$.

Denote

$$
\begin{aligned}
\mathbf{G}(\mathbf{x})= & {\left[\begin{array}{ccc}
L_{\mathbf{g}_{1}} L_{\mathbf{f}}^{r_{1}-1} h_{1}(\mathbf{x}) & \cdots & L_{\mathbf{g}_{m}} L_{\mathbf{f}}^{r_{1}-1} h_{1}(\mathbf{x}) \\
L_{\mathbf{g}_{1}} L_{\mathbf{f}}^{r_{2}-1} h_{2}(\mathbf{x}) & \cdots & L_{\mathbf{g}_{m}} L_{\mathbf{f}}^{r_{2}-1} h_{2}(\mathbf{x}) \\
\cdots & \ddots & \cdots \\
L_{\mathbf{g}_{1}} L_{\mathbf{f}}^{r_{m}-1} h_{m}(\mathbf{x}) & \cdots & L_{\mathbf{g}_{m}} L_{\mathbf{f}}^{r_{m}-1} h_{m}(\mathbf{x})
\end{array}\right] } \\
= & {\left[\begin{array}{ccc}
g_{11}(\mathbf{x}) & \cdots & g_{1 m}(\mathbf{x}) \\
\vdots & \ddots & \vdots \\
g_{m 1}(\mathbf{x}) & \cdots & g_{m m}(\mathbf{x})
\end{array}\right] . }
\end{aligned}
$$


Note that $\mathbf{G}(\mathbf{x})$ is nonsingular at $\mathbf{x}=\mathbf{x}_{0}$.

Using feedback linearization, the nonlinear system (1) can be transformed into the following form [43]:

$$
\mathbf{y}^{(\mathbf{r})}=\mathbf{F}(\mathbf{x})+\mathbf{G}(\mathbf{x}) \mathbf{u}+\mathbf{d}
$$

where

$$
\begin{gathered}
\mathbf{y}^{(\mathbf{r})}=\left[y_{1}^{\left(r_{1}\right)}, y_{2}^{\left(r_{2}\right)}, \ldots, y_{m}^{\left(r_{m}\right)}\right]^{T}, \\
\mathbf{F}(\mathbf{x})=\left[\begin{array}{c}
L_{\mathbf{f}}^{r_{1}} h_{1}(\mathbf{x}) \\
L_{\mathbf{f}}^{r_{2}} h_{2}(\mathbf{x}) \\
\vdots \\
L_{\mathbf{f}}^{r_{m}} h_{m}(\mathbf{x})
\end{array}\right]=\left[\begin{array}{c}
f_{1}(\mathbf{x}) \\
f_{2}(\mathbf{x}) \\
\vdots \\
f_{m}(\mathbf{x})
\end{array}\right] \\
\mathbf{d}=\left[\begin{array}{c}
\sum_{i=1}^{r_{1}} L_{\dot{\mathbf{x}}}^{i-1} L_{d^{\prime}} L_{\mathbf{f}}^{r_{1}-i} h_{1}(\mathbf{x}) \\
\sum_{i=1}^{r_{2}} L_{\dot{\mathbf{x}}}^{i-1} L_{d^{\prime}} L_{\mathbf{f}}^{r_{2}-i} h_{2}(\mathbf{x}) \\
\vdots \\
\mathbf{x}=\left[y_{1}, \ldots, y_{1}^{\left(r_{1}\right)}, y_{2}, \ldots, y_{2}^{\left(r_{2}\right)}, \ldots, y_{m}, \ldots, y_{m}^{\left(r_{m}\right)}\right]
\end{array}\right] .\left[\begin{array}{c}
d_{1} \\
d_{2} \\
\vdots \\
\sum_{i=1}^{r_{m}} L_{\dot{\mathbf{x}}}^{i-1} L_{d^{\prime}} L_{\mathbf{f}}^{r_{m}-i} h_{m}(\mathbf{x})
\end{array}\right] .
\end{gathered}
$$

In system (4), d still represents unknown bounded external disturbance vector. The relative degree of the system is assumed to be equal to the order of the system and is expressed as $\sum_{i=1}^{m} r_{i}=n$, which implies that the system does not have any zero dynamics.

Let the desired output trajectory be given by

$$
\mathbf{y}_{d}=\left[y_{d 1}, y_{d 2}, \ldots, y_{d m}\right]^{T} \text {. }
$$

This paper aims to develop a robust adaptive fuzzy tracking control scheme such that all closed-loop system signals asymptotically converge to a compact set in the presence of input saturation, system uncertainties, and external disturbances and ensure the system outputs $\mathbf{y}$ track the desired trajectory $\mathbf{y}_{d}$. For system (4) to be controllable, the following assumptions are made.

Assumption 1. The desired trajectory $y_{d i}$ and its $n$th order derivatives are known and bounded.

Assumption 2. For $\mathbf{x}$ in certain controllability region $\mathbf{U}_{c} \in R^{n}$, $\sigma(\mathbf{G}(\mathbf{x})) \neq 0$, where $\sigma(\mathbf{G}(\mathbf{x}))$ denotes the minimum singular value of the matrix $\mathbf{G}(\mathbf{x})$.

2.2. Fuzzy Logic Systems. In this paper, fuzzy logic systems are used to approximate unknown nonlinear functions $\mathbf{F}(\mathbf{x})$ and $\mathbf{G}(\mathbf{x})$. Following, the approximation presentation of fuzzy logic systems is given. Without loss of generality, the unknown uncertainty is assumed as $f(\mathbf{x})$. A fuzzy logic system consists of four parts: the knowledge base, the fuzzifier, the fuzzy inference engine working on fuzzy rules, and the defuzzifier. The fuzzy inference engine uses fuzzy IF-THEN rules to perform a mapping from an input linguistic vector $\mathbf{x}=\left[x_{1}, x_{2}, \ldots, x_{n}\right]^{T} \in R^{n}$ to an output variable $y \in R$. Then, the $i$ th fuzzy rule can be represented as [3]

$$
\begin{aligned}
& R^{l}: \text { If } x_{1} \text { is } F_{1}^{l} \text { and } \cdots \text { and } x_{n} \text { is } F_{n}^{l} \text {, then } y \text { is } G^{l} \\
& (l=1,2, \ldots, r),
\end{aligned}
$$

where $F_{i}^{l}$ and $G^{l}$ are fuzzy sets characterized with the fuzzy membership functions $\mu_{F_{i}^{l}}\left(x_{i}\right)$ and $\mu_{G}(y)$, respectively, and $r$ is the number of fuzzy rules.

Through singleton function, center average defuzzifier, and product inference, the fuzzy logic system can be expressed as follows:

$$
y(\mathbf{x})=\frac{\sum_{l=1}^{r} \bar{y}^{l}\left(\prod_{i=1}^{n} \mu_{F_{i}^{l}}\left(x_{i}\right)\right)}{\sum_{l=1}^{r} \prod_{i=1}^{n} \mu_{F_{i}^{l}}\left(x_{i}\right)},
$$

where $\bar{y}^{l}=\max _{y \in R} \mu_{G^{l}}(y)$.

By introducing the concept of fuzzy basis function vector, the final output of the fuzzy logic system can be expressed as

$$
y(\mathbf{x})=\boldsymbol{\theta}^{T} \boldsymbol{\xi}(\mathbf{x}),
$$

where $\boldsymbol{\theta}=\left[\bar{y}^{1}, \ldots, \bar{y}^{r}\right]^{T}$ is the adjustable parameter vector and $\boldsymbol{\xi}(\mathbf{x})=\left[\xi_{1}(\mathbf{x}), \ldots, \xi_{r}(\mathbf{x})\right]^{T}$ is the fuzzy basis function vector. The fuzzy basis function is defined as follows:

$$
\xi_{l}(\mathbf{x})=\frac{\sum_{l=1}^{r}\left(\prod_{i=1}^{n} \mu_{F_{i}^{l}}\left(x_{i}\right)\right)}{\sum_{l=1}^{r} \prod_{i=1}^{n} \mu_{F_{i}^{l}}\left(x_{i}\right)} .
$$

Lemma 3. Let $f(\mathbf{x})$ be a continuous function defined on a compact set $\mathbf{U}_{c}$. Then, for any constants $\varepsilon>0$, there exists a fuzzy logic system such as [2]

$$
\sup _{\mathbf{x} \in \mathbf{U}_{c}}\left|f(\mathbf{x})-\boldsymbol{\theta}^{T} \boldsymbol{\xi}(\mathbf{x})\right| \leq \varepsilon .
$$

Define the optimal parameter vector $\boldsymbol{\theta}^{*}$ as

$$
\boldsymbol{\theta}^{*}=\arg \min _{\boldsymbol{\theta} \in \boldsymbol{\Omega}_{f}}\left[\sup _{\mathbf{x} \in \mathbf{U}_{c}}\left|f(\mathbf{x})-\boldsymbol{\theta}^{T} \boldsymbol{\xi}(\mathbf{x})\right|\right] .
$$

Under the optimization parameter vector, the unknown function $f(\mathbf{x})$ can be written as

$$
f(\mathbf{x})=\boldsymbol{\theta}^{* T} \boldsymbol{\xi}(\mathbf{x})+\varepsilon,
$$

where $\varepsilon$ is the fuzzy minimum approximation error.

In this work, $\mathbf{F}(\mathbf{x})$ and $\mathbf{G}(\mathbf{x})$ are unknown vector and matrix, respectively, and the fuzzy logic systems are used to approximate the unknown functions. Let $f_{i}(\mathbf{x})(i=1, \ldots, m)$ and $g_{i j}(\mathbf{x})(i, j=1, \ldots, m)$ be approximated by fuzzy logic systems as follows:

$$
\begin{gathered}
\widehat{f}_{i}\left(\mathbf{x} \mid \boldsymbol{\theta}_{f_{i}}\right)=\boldsymbol{\theta}_{f_{i}}^{T} \boldsymbol{\xi}_{f_{i}}(\mathbf{x}) \quad i=1, \ldots, m \\
\widehat{g}_{i j}\left(\mathbf{x} \mid \boldsymbol{\theta}_{g_{i j}}\right)=\boldsymbol{\theta}_{g_{i j}}^{T} \boldsymbol{\xi}_{g_{i j}}(\mathbf{x}) \quad i, j=1, \ldots, m,
\end{gathered}
$$


where $\boldsymbol{\theta}_{f_{i}} \in \mathbf{R}^{M_{f_{i}}}$ and $\boldsymbol{\theta}_{g_{i j}} \in \mathbf{R}^{M_{g_{i j}}}$ are parameter vectors, $\boldsymbol{\xi}_{f_{i}}(\mathbf{x}) \in \mathbf{R}^{M_{f_{i}}}$ and $\boldsymbol{\xi}_{g_{i j}}(\mathbf{x}) \in \mathbf{R}^{M_{g_{i j}}}$ are fuzzy basis function vectors, and $M_{f_{i}}$ and $M_{g_{i j}}$ are the corresponding dimensions of the basis vectors.

Denote

$$
\begin{gathered}
\widehat{\mathbf{F}}\left(\mathbf{x} \mid \boldsymbol{\theta}_{F}\right)=\left[\begin{array}{c}
\widehat{f}_{1}\left(\mathbf{x} \mid \boldsymbol{\theta}_{f_{1}}\right) \\
\vdots \\
\widehat{f}_{m}\left(\mathbf{x} \mid \boldsymbol{\theta}_{f_{m}}\right)
\end{array}\right] \\
\widehat{\mathbf{G}}\left(\mathbf{x} \mid \boldsymbol{\theta}_{G}\right)=\left[\begin{array}{ccc}
\widehat{g}_{11}\left(\mathbf{x} \mid \boldsymbol{\theta}_{g_{11}}\right) & \cdots & \widehat{g}_{1 m}\left(\mathbf{x} \mid \boldsymbol{\theta}_{g_{1 m}}\right) \\
\vdots & \ddots & \vdots \\
\widehat{g}_{m 1}\left(\mathbf{x} \mid \boldsymbol{\theta}_{g_{m 1}}\right) & \cdots & \widehat{g}_{m m}\left(\mathbf{x} \mid \boldsymbol{\theta}_{g_{m m}}\right)
\end{array}\right] .
\end{gathered}
$$

Then $\widehat{\mathbf{F}}\left(\mathbf{x} \mid \boldsymbol{\theta}_{F}\right)$ and $\widehat{\mathbf{G}}\left(\mathbf{x} \mid \boldsymbol{\theta}_{G}\right)$ can be used as the approximation of $\mathbf{F}(\mathbf{x})$ and $\mathbf{G}(\mathbf{x})$, respectively.

Define the optimal approximation weight vectors for $f_{i}(i=1, \ldots, m)$ and $g_{i j}(i, j=1, \ldots, m)$ as follows:

$$
\begin{gathered}
\boldsymbol{\theta}_{f_{i}}^{*}=\arg \min _{\boldsymbol{\theta}_{f_{i}} \in \boldsymbol{\Omega}_{\mathbf{F}}}\left\{\sup _{\mathbf{x} \in \mathbf{U}_{c}}\left|\widehat{f}_{i}\left(\mathbf{x} \mid \boldsymbol{\theta}_{f_{i}}\right)-f_{i}(\mathbf{x})\right|\right\} \\
\boldsymbol{\theta}_{g_{i j}}^{*}=\arg \min _{\boldsymbol{\theta}_{g_{i j}} \in \boldsymbol{\Omega}_{\mathbf{G}}}\left\{\sup _{\mathbf{x} \in \mathbf{U}_{c}}\left|\widehat{g}_{i j}\left(\mathbf{x} \mid \boldsymbol{\theta}_{g_{i j}}\right)-g_{i j}(\mathbf{x})\right|\right\},
\end{gathered}
$$

where $\boldsymbol{\Omega}_{\mathrm{F}}, \boldsymbol{\Omega}_{\mathrm{G}}$, and $\mathrm{U}_{c}$ denote the sets of suitable bounds on $\boldsymbol{\theta}_{f_{i}}, \boldsymbol{\theta}_{g_{i j}}$, and $\mathbf{x}$ respectively. $\boldsymbol{\theta}_{f_{i}}^{*}(i=1, \ldots, m)$ and $\boldsymbol{\theta}_{g_{i j}}^{*}(i, j=$ $1, \ldots, m)$ are constants vectors.

The unknown function $f_{i}$ and $g_{i j}$ can be expressed as

$$
\begin{gathered}
f_{i}(\mathbf{x})=\boldsymbol{\theta}_{f_{i}}^{* T} \boldsymbol{\xi}_{f_{i}}(\mathbf{x})+\varepsilon_{f_{i}} \\
g_{i j}(\mathbf{x})=\boldsymbol{\theta}_{g_{i j}}^{* T} \boldsymbol{\xi}_{g_{i j}}(\mathbf{x})+\varepsilon_{g_{i j}} \quad i, j=1, \ldots, m,
\end{gathered}
$$

where $\varepsilon_{f_{i}}$ and $\varepsilon_{g_{i j}}$ are the smallest approximation errors of the fuzzy logic systems.

\section{Adaptive Fuzzy Robust Control Designs}

In this section, we first design the adaptive fuzzy approximation based control problem without control saturation and then consider the case where actuators have physical limitations, such as magnitude and rate constraints.

3.1. Adaptive Fuzzy Robust Control. The tracking errors are defined as

$$
e_{i}=y_{d i}-y_{i} \quad i=1,2, \ldots, m .
$$

Define $u_{l i}(i=1, \ldots, m)$ as follows:

$$
u_{l i}=y_{d i}^{\left(r_{i}\right)}+\sum_{j=1}^{r_{i}} k_{i j} e_{i}^{(j-1)} \quad i=1, \ldots, m,
$$

where $k_{i 1}, \ldots, k_{i r_{i}}$ are parameters to be chosen such that the roots of the equation $s^{r_{i}}+k_{i r_{i}} s^{r_{i}-1}+\cdots+k_{i 1}=0$ in the open left-half complex plane.

If $\mathbf{F}(\mathbf{x})$ and $\mathbf{G}(\mathbf{x})$ are known, external disturbances are ignored; then, according to nonlinear dynamic inversion control techniques, the control law is given by

$$
\mathbf{u}=\mathbf{G}^{-1}(\mathbf{x})\left[-\mathbf{F}(\mathbf{x})+\mathbf{u}_{l}\right],
$$

where $\mathbf{u}_{l}=\left[u_{l 1}, \ldots, u_{l m}\right]^{T}$.

Because $\mathbf{F}(\mathbf{x})$ and $\mathbf{G}(\mathbf{x})$ are unknown, $d_{i} \neq 0$, the control law (23) cannot be implemented in practice. Using the approximation of $\mathbf{F}(\mathbf{x})$ and $\mathbf{G}(\mathbf{x})$, and considering external disturbances, the controller is modified as follows:

$$
\mathbf{u}=\widehat{\mathbf{G}}^{-1}\left(\mathbf{x} \mid \boldsymbol{\theta}_{G}\right)\left[-\widehat{\mathbf{F}}\left(\mathbf{x} \mid \boldsymbol{\theta}_{F}\right)+\mathbf{u}_{l}+\mathbf{u}_{d}\right],
$$

where $\mathbf{u}_{d}$ is the robust compensation term, which is used to attenuate the effect of external disturbances and approximation errors.

Substituting (24) into system (4) yields

$$
\begin{aligned}
\mathbf{y}^{(r)}= & \mathbf{F}(\mathbf{x})-\widehat{\mathbf{F}}\left(\mathbf{x} \mid \boldsymbol{\theta}_{F}\right)+\left[\mathbf{G}(\mathbf{x})-\widehat{\mathbf{G}}\left(\mathbf{x} \mid \boldsymbol{\theta}_{G}\right)\right] \mathbf{u}+\mathbf{u}_{l} \\
& +\mathbf{u}_{d}+\mathbf{d} .
\end{aligned}
$$

Considering (22), the $i$ th subsystem of (25) can be rewritten as

$$
\begin{aligned}
\dot{\mathbf{e}}_{i}=\mathbf{A}_{i} \mathbf{e}_{i}+\mathbf{B}_{i}\{[ & \left.\widehat{f}_{i}\left(\mathbf{x} \mid \boldsymbol{\theta}_{f_{i}}\right)-f_{i}(\mathbf{x})\right] \\
& \left.+\sum_{j=1}^{m}\left[\widehat{g}_{i j}\left(\mathbf{x} \mid \boldsymbol{\theta}_{g_{i j}}\right)-g_{i j}(\mathbf{x})\right] u_{j}\right\}
\end{aligned}
$$

$$
-\mathbf{B}_{i} u_{d i}-\mathbf{B}_{i} d_{i},
$$

where $u_{d i}$ is the $i$ th element of $\mathbf{u}_{d}$ and

$$
\begin{gathered}
\mathbf{A}_{i}=\left[\begin{array}{ccccc}
0 & 1 & 0 & \cdots & 0 \\
0 & 0 & 1 & \cdots & 0 \\
\vdots & \vdots & \vdots & \ddots & \vdots \\
0 & 0 & 0 & \cdots & 1 \\
-k_{i 1} & -k_{i 2} & -k_{i 3} & \cdots & -k_{i r_{i}}
\end{array}\right] \quad \mathbf{B}_{i}=\left[\begin{array}{c}
0 \\
0 \\
\vdots \\
0 \\
1
\end{array}\right] \\
\mathbf{e}_{i}=\left[\begin{array}{c}
e_{i} \\
\dot{e}_{i} \\
\vdots \\
e_{i}^{\left(r_{i}-2\right)} \\
e_{i}^{\left(r_{i}-1\right)}
\end{array}\right] .
\end{gathered}
$$

Define the minimum approximation error as

$$
\omega_{i}^{\prime}=\left[\widehat{f}_{i}\left(\mathbf{x} \mid \boldsymbol{\theta}_{f_{i}}^{*}\right)-f_{i}(\mathbf{x})\right]+\sum_{j=1}^{m}\left[\widehat{g}_{i j}\left(\mathbf{x} \mid \boldsymbol{\theta}_{g_{i j}}^{*}\right)-g_{i j}(\mathbf{x})\right] u_{j} .
$$


According to fuzzy system theory, the following assumption is reasonable.

Assumption 4. The minimum approximation error is square integrable, that is, $\int_{0}^{T} \boldsymbol{\omega}_{i}^{\prime T} \boldsymbol{\omega}_{i}^{\prime} d t<\infty$.

Using the definition (28) and the optimal approximation for $f_{i}(\mathbf{x})$ and $g_{i j}(\mathbf{x}),(26)$ can be rewritten in the following form:

$$
\begin{aligned}
\dot{\mathbf{e}}_{i}= & \mathbf{A}_{i} \mathbf{e}_{i}+\mathbf{B}_{i}\left[\tilde{\boldsymbol{\theta}}_{f_{i}}^{T} \boldsymbol{\xi}_{f_{i}}(\mathbf{x})+\sum_{j=1}^{m} \widetilde{\boldsymbol{\theta}}_{g_{i j}}^{T} \boldsymbol{\xi}_{g_{i j}}(\mathbf{x}) u_{j}\right] \\
& -\mathbf{B}_{i} u_{d i}+\mathbf{B}_{i} \omega_{i},
\end{aligned}
$$

where $\widetilde{\boldsymbol{\theta}}_{f_{i}}=\boldsymbol{\theta}_{f_{i}}-\boldsymbol{\theta}_{f_{i}}^{*}, \widetilde{\boldsymbol{\theta}}_{g_{i j}}=\boldsymbol{\theta}_{g_{i j}}-\boldsymbol{\theta}_{g_{i j}}^{*}$, and $\omega_{i}=\omega_{i}^{\prime}-d_{i}$.

Theorem 5. Consider the uncertain MIMO nonlinear system presented by (4) with external disturbance. If the controller is chosen as (24), the parameters update laws and $u_{d i}$ are adopted as follows:

$$
\begin{gathered}
\dot{\boldsymbol{\theta}}_{f_{i}}=-\gamma_{f_{i}} \boldsymbol{\xi}_{f_{i}}(\mathbf{x}) \mathbf{B}_{i}^{T} \mathbf{P}_{i} \mathbf{e}_{i} \quad i=1, \ldots, m \\
\dot{\boldsymbol{\theta}}_{g_{i j}}=-\gamma_{g_{i j}} \boldsymbol{\xi}_{g_{i j}}(\mathbf{x}) \mathbf{B}_{i}^{T} \mathbf{P}_{i} \mathbf{e}_{i} u_{j} \quad i, j=1, \ldots, m \\
u_{d i}=\frac{1}{2 \rho_{i}^{2}} \mathbf{B}_{i}^{T} \mathbf{P}_{i} \mathbf{e}_{i} \quad i=1, \ldots, m .
\end{gathered}
$$

Then the following $H_{\infty}$ tracking performance can be obtained

$$
\begin{aligned}
\int_{0}^{T} \mathbf{e}^{T} \mathbf{Q e} d t \leq & \mathbf{e}^{T}(0) \mathbf{P e}(0)+\sum_{i=1}^{m} \frac{1}{\gamma_{f_{i}}} \widetilde{\boldsymbol{\theta}}_{f_{i}}^{T}(0) \widetilde{\boldsymbol{\theta}}_{f_{i}}(0) \\
& +\sum_{i, j=1}^{m} \frac{1}{\gamma_{g_{i j}}} \widetilde{\boldsymbol{\theta}}_{g_{i j}}^{T}(0) \widetilde{\boldsymbol{\theta}}_{g_{i j}}(0)+\sum_{i=1}^{m} \rho_{i}^{2} \int_{0}^{T} \omega_{i}^{2} d t,
\end{aligned}
$$

where $\gamma_{f_{i}}(i=1, \ldots, m)$ and $\gamma_{g_{i j}}(i, j=1, \ldots, m)$ are positive adaptive scalar, $\rho_{i}(i=1, \ldots, m)$ are positive parameters representing for prescribed disturbance attenuation levels, $\mathbf{e}=\left[\mathbf{e}_{1}^{T}, \ldots, \mathbf{e}_{m}^{T}\right]^{T}, \mathbf{Q}=\operatorname{diag}\left[\mathbf{Q}_{1}, \ldots, \mathbf{Q}_{m}\right]$, and $\mathbf{Q}_{i} \in$ $\mathbf{R}^{m \times m}(i=1, \ldots, m)$ are arbitrary symmetric positive definite matrices, and $\mathbf{P}=\operatorname{diag}\left[\mathbf{P}_{1}, \ldots, \mathbf{P}_{m}\right]$ and $\mathbf{P}_{i} \in \mathbf{R}^{m \times m}(i=$ $1, \ldots, m)$ are symmetric positive definite solution of the following equations:

$$
\mathbf{P}_{i} \mathbf{A}_{i}+\mathbf{A}_{i}^{T} \mathbf{P}_{i}=-\mathbf{Q}_{i},
$$

where $\mathbf{Q}_{i} \in \mathbf{R}^{m \times m}(i=1, \ldots, m)$ are arbitrary symmetric positive definite matrices.

Proof. For the $i$ th subsystem of (4), consider the following Lyapunov function candidate

$$
V_{i}=\frac{1}{2} \mathbf{e}_{i}^{T} \mathbf{P}_{i} \mathbf{e}_{i}+\frac{1}{2 \gamma_{f_{i}}} \widetilde{\boldsymbol{\theta}}_{f_{i}}^{T} \widetilde{\boldsymbol{\theta}}_{f_{i}}+\frac{1}{2} \sum_{i=1}^{m} \frac{1}{\gamma_{g_{i j}}} \widetilde{\boldsymbol{\theta}}_{g_{i j}}^{T} \widetilde{\boldsymbol{\theta}}_{g_{i j}} .
$$

Differentiating (35) and considering (29) yield

$$
\begin{aligned}
\dot{V}_{i}= & \frac{1}{2} \dot{\mathbf{e}}_{i}^{T} \mathbf{P}_{i} \mathbf{e}_{i}+\frac{1}{2} \mathbf{e}_{i}^{T} \mathbf{P}_{i} \dot{\mathbf{e}}_{i}+\frac{1}{\gamma_{f_{i}}} \dot{\tilde{\boldsymbol{\theta}}}_{f_{i}}^{T} \widetilde{\boldsymbol{\theta}}_{f_{i}}+\sum_{i=1}^{m} \frac{1}{\gamma_{g_{i j}}} \dot{\tilde{\boldsymbol{\theta}}}_{g_{i j}}^{T} \widetilde{\boldsymbol{\theta}}_{g_{i j}} \\
= & \frac{1}{2} \mathbf{e}_{i}^{T}\left(\mathbf{P}_{i} \mathbf{A}_{i}+\mathbf{A}_{i}^{T} \mathbf{P}_{i}\right) \mathbf{e}_{i}+\frac{1}{2}\left(-u_{d i}+\omega_{i}\right)\left(\mathbf{B}_{i}^{T} \mathbf{P}_{i} \mathbf{e}_{i}+\mathbf{e}_{i}^{T} \mathbf{P}_{i} \mathbf{B}_{i}\right) \\
& +\frac{1}{\gamma_{f_{i}}}\left[\gamma_{f_{i}} \mathbf{e}_{i}^{T} \mathbf{P}_{i} \mathbf{B}_{i} \boldsymbol{\xi}_{f_{i}}^{T}(\mathbf{x})+\dot{\tilde{\boldsymbol{\theta}}}_{f_{i}}^{T}\right] \widetilde{\boldsymbol{\theta}}_{f_{i}} \\
& +\sum_{i=1}^{m} \frac{1}{\gamma_{g_{i j}}}\left[\gamma_{g_{i j}} \mathbf{e}_{i}^{T} \mathbf{P}_{i} \mathbf{B}_{i} \boldsymbol{\xi}_{g_{i j}}^{T}(\mathbf{x}) u_{j}+\dot{\tilde{\boldsymbol{\theta}}}_{g_{i j}^{T}}^{T}\right] \widetilde{\boldsymbol{\theta}}_{g_{i j}}
\end{aligned}
$$

Substituting (30)-(32) into (36), we obtain

$$
\begin{aligned}
\dot{V}_{i} & =-\frac{1}{2} \mathbf{e}_{i}^{T} \mathbf{Q}_{i} \mathbf{e}_{i}+\frac{1}{2} \omega_{i}\left(\mathbf{B}_{i}^{T} \mathbf{P}_{i} \mathbf{e}_{i}+\mathbf{e}_{i}^{T} \mathbf{P}_{i} \mathbf{B}_{i}\right)-\frac{1}{2 \rho_{i}^{2}} \mathbf{e}_{i}^{T} \mathbf{P}_{i} \mathbf{B}_{i} \mathbf{B}_{i}^{T} \mathbf{P}_{i} \mathbf{e}_{i} \\
& =-\frac{1}{2} \mathbf{e}_{i}^{T} \mathbf{Q}_{i} \mathbf{e}_{i}+\frac{1}{2} \rho_{i}^{2} \omega_{i}^{2}-\frac{1}{2}\left(\frac{1}{\rho_{i}} \mathbf{e}_{i}^{T} \mathbf{P}_{i} \mathbf{B}_{i}-\rho_{i} \omega_{i}\right)^{2} \\
& \leq-\frac{1}{2} \mathbf{e}_{i}^{T} \mathbf{Q}_{i} \mathbf{e}_{i}+\frac{1}{2} \rho_{i}^{2} \omega_{i}^{2} .
\end{aligned}
$$
yields

Integrating both sides of the above inequality from 0 to $T$

$$
\frac{1}{2} \int_{0}^{T} \mathbf{e}_{i}^{T} \mathbf{Q}_{i} \mathbf{e}_{i} d t \leq V_{i}(0)-V_{i}(T)+\frac{\rho_{i}^{2}}{2} \int_{0}^{T} \omega_{i}^{2} d t .
$$

Since $V_{i}(T)$ is nonnegative, according to the definition of $V_{i}$, the following inequality is obtained:

$$
\begin{aligned}
\int_{0}^{T} \mathbf{e}_{i}^{T} \mathbf{Q}_{i} \mathbf{e}_{i} d t \leq & \mathbf{e}_{i}^{T}(0) \mathbf{P}_{i} \mathbf{e}_{i}(0)+\frac{1}{\gamma_{f_{i}}} \widetilde{\boldsymbol{\theta}}_{f_{i}}^{T}(0) \tilde{\boldsymbol{\theta}}_{f_{i}}(0) \\
& +\sum_{i=1}^{m} \frac{1}{\gamma_{g_{i j}}} \widetilde{\boldsymbol{\theta}}_{g_{i j}}^{T}(0) \widetilde{\boldsymbol{\theta}}_{g_{i j}}(0)+\rho_{i}^{2} \int_{0}^{T} \omega_{i}^{2} d t .
\end{aligned}
$$

Considering the Lyapunov function $V=\sum_{i=1}^{m} V_{i}$, it is easy to obtain the $H_{\infty}$ tracking performance index (55). This completes the proof.

The controller proposed by (24) is able to guarantee the Lyapunov stability of the closed-loop system and attenuate the effect of system uncertainties and external disturbances. However, no saturation on actuators is taken into account, which is rather important for practical applications (including satellite systems). Assume that the control input $\mathbf{u}$ is constraint by saturation functions; it can be readily shown that the control law (24), the parameters update laws (30), (31), and the robust compensation term (32) with saturation limits cannot guarantee the stability of the closed-loop system.

It is expected that during saturation the magnitude of the tracking error will increase, since the control signal is not being achieved. This tracking error is not the result of function approximation error; therefore, we need to be careful so 
that the approximator does not cause "unlearning" during the period when the actuators are saturated. Clearly, the parameters update laws (30) and (31) depend on the tracking error $\mathbf{e}_{i}$, thus if the tracking error increases due to saturation, the parameters update laws may cause a significant change in the weights in response to the increase in tracking error. Next, we develop a robust fuzzy adaptive control scheme to address the input saturation problem.

3.2. Adaptive Fuzzy Control with Input Saturation. When the actuators have physical constraints such as the magnitude and rate limitations, the above approach may not be able to be successfully implemented. Considering the magnitude and rate limitations on the actuator, controller (24) is modified as

$$
\begin{aligned}
\mathbf{u}_{c} & =\widehat{\mathbf{G}}^{-1}\left(\mathbf{x} \mid \boldsymbol{\theta}_{G}\right)\left[-\widehat{\mathbf{F}}\left(\mathbf{x} \mid \boldsymbol{\theta}_{F}\right)+\mathbf{u}_{l}+\mathbf{u}_{a w}+\mathbf{u}_{d}\right] \\
\mathbf{u} & =S_{M R}\left(\mathbf{u}_{c}\right),
\end{aligned}
$$

where $\mathbf{u}_{c}$ is obtained by certainty equivalence principle; $\mathbf{u}_{a w}$ is the auxiliary control term which is used to compensate the effect of input saturation; $S_{M R}(\cdot)$ is a function including the magnitude and rate constraints which can produce a limited output.

The state space representation of each component of $\mathbf{u}_{c}$ is

$$
\begin{aligned}
& {\left[\begin{array}{c}
\dot{r}_{i 1} \\
\dot{r}_{i 2}
\end{array}\right]=\left[\begin{array}{c}
r_{i 2} \\
S_{R}\left(\omega_{i}\left(S_{M}\left(u_{c i}\right)-r_{i 1}\right)\right)
\end{array}\right]} \\
& {\left[\begin{array}{c}
u_{i} \\
\dot{u}_{i}
\end{array}\right]=\left[\begin{array}{c}
r_{i 1} \\
r_{i 2}
\end{array}\right],}
\end{aligned}
$$

where $u_{c i}$ is the $i$ th element of $\mathbf{u}_{c}$, and $u_{i}$ is the $i$ th element of $\mathbf{u}$; $\omega_{i}$ is the natural frequency; and $S_{M}(\cdot)$ and $S_{R}(\cdot)$ are the saturation functions corresponding to magnitude and rate, respectively. The function $S_{M}(\cdot)$ is defined as

$$
S_{M}(\cdot)= \begin{cases}M & \text { if } x \geq M \\ x & \text { if }|x|<M \\ -M & \text { if } x \leq-M\end{cases}
$$

and $S_{R}(\cdot)$ has the same definition.

To compensate the effects of input limitations, an auxiliary system is introduced as follows [35]:

$$
\begin{gathered}
\dot{\xi}_{i 1}=\xi_{i 2}-\lambda_{i 1} \xi_{i 1} \ldots \dot{\xi}_{i, r_{i}-1}=\xi_{i r_{i}}-\lambda_{i, r_{i}-1} \xi_{i, r_{i}-1} \\
\dot{\xi}_{i r_{i}}=-\lambda_{i r_{i}} \xi_{i r_{i}}+\sum_{j=1}^{m} \widehat{g}_{i j}\left(\mathbf{x} \mid \boldsymbol{\theta}_{g_{i j}}\right)\left(u_{j}-u_{c j}\right) \quad i=1,2, \ldots, m,
\end{gathered}
$$

where $\lambda_{i j}\left(i=1, \ldots, m ; j=1, \ldots, r_{i}\right)$ are positive design parameters.
Define the modified tracking error as

$$
\bar{e}_{i}=y_{d i}-y_{i}-\xi_{i 1} \quad i=1,2, \ldots, m
$$

From (4), (40), and (43), we obtain

$$
\begin{aligned}
& \bar{e}_{i}^{\left(r_{i}\right)}+\sum_{j=1}^{r_{i}} k_{i j} \bar{e}_{i}^{\left(r_{i}-1\right)} \\
& =\widehat{f}_{i}\left(\mathbf{x} \mid \boldsymbol{\theta}_{f_{i}}\right)-f_{i}(\mathbf{x})+\sum_{j=1}^{m}\left[\widehat{g}_{i j}\left(\mathbf{x} \mid \boldsymbol{\theta}_{g_{i j}}\right)-g_{i j}(\mathbf{x})\right] u_{j} \\
& \quad-u_{a w i}-u_{d i}-d_{i}-\xi_{i 1}^{\left(r_{i}\right)}-\sum_{j=1}^{r_{i}} k_{i j} \xi_{i 1}^{(j-1)} \\
& \quad+\sum_{j=1}^{m} \widehat{g}_{i j}\left(\mathbf{x} \mid \boldsymbol{\theta}_{g_{i j}}\right)\left(u_{j}-u_{c j}\right),
\end{aligned}
$$

where $u_{a w i}$ is the $i$ th element of $\mathbf{u}_{a w}$.

From (43), the term $\xi_{i 1}^{\left(r_{i}\right)}$ can be expressed as

$$
\xi_{i 1}^{\left(r_{i}\right)}=-\sum_{j=1}^{r_{i}} \lambda_{i j} \xi_{i j}^{\left(r_{i}-j\right)}+\sum_{j=1}^{m} \widehat{g}_{i j}\left(\mathbf{x} \mid \boldsymbol{\theta}_{g_{i j}}\right)\left(u_{j}-u_{c j}\right)
$$

Let

$$
u_{a w i}=\sum_{j=1}^{r_{i}}\left(\lambda_{i j} \xi_{i j}^{\left(r_{i}-j\right)}-k_{i j} \xi_{i j}^{(j-1)}\right)
$$

With (46) and (47), (45) becomes

$$
\begin{aligned}
\bar{e}_{i}^{\left(r_{i}\right)}+\sum_{j=1}^{r_{i}} k_{i j} \bar{e}_{i}^{\left(r_{i}-1\right)} \\
=\widehat{f}_{i}\left(\mathbf{x} \mid \boldsymbol{\theta}_{f_{i}}\right)-f_{i}(\mathbf{x})+\sum_{j=1}^{m}\left[\widehat{g}_{i j}\left(\mathbf{x} \mid \boldsymbol{\theta}_{g_{i j}}\right)-g_{i j}(\mathbf{x})\right] u_{j} \\
\quad-u_{d i}-d_{i} .
\end{aligned}
$$

Define $\overline{\mathbf{e}}_{i}=\left[\bar{e}_{i}, \dot{\bar{e}}_{i}, \ldots, \bar{e}_{i}^{\left(r_{i}-1\right)}\right]^{T}$ and using the optimal approximation for $f_{i}(\mathbf{x})$ and $g_{i j}(\mathbf{x}),(48)$ can be rewritten as

$$
\dot{\overline{\mathbf{e}}}{ }_{i}=\mathbf{A}_{i} \overline{\mathbf{e}}_{i}+\mathbf{B}_{i}\left[\widetilde{\boldsymbol{\theta}}_{f_{i}}^{T} \boldsymbol{\xi}_{f_{i}}(\mathbf{x})+\sum_{j=1}^{m} \widetilde{\boldsymbol{\theta}}_{g_{i j}}^{T} \boldsymbol{\xi}_{g_{i j}}(\mathbf{x}) u_{j}\right]-\mathbf{B}_{i} u_{d i}+\mathbf{B}_{i} \omega_{i} .
$$

Theorem 6. Consider the uncertain MIMO nonlinear system presented by (4) with external disturbance and input saturation, if the controller is chosen as (40), the auxiliary control 
term $\mathbf{u}_{a w}$, the parameters update laws, and $u_{d i}$ are adopted as follows:

$$
\begin{gathered}
\dot{\xi}_{i 1}=\xi_{i 2}-\lambda_{i 1} \xi_{i 1} \ldots \dot{\xi}_{i, r_{i}-1}=\xi_{i r_{i}}-\lambda_{i, r_{i}-1} \xi_{i, r_{i}-1} \\
\dot{\xi}_{i r_{i}}=-\lambda_{i r_{i}} \xi_{i r_{i}}+\sum_{j=1}^{m} \widehat{g}_{i j}\left(\mathbf{x} \mid \boldsymbol{\theta}_{g_{i j}}\right)\left(u_{j}-u_{c j}\right) \quad i=1,2, \ldots, m \\
\mathbf{u}_{a w}=\left[\sum_{j=1}^{r_{1}}\left(\lambda_{1 j} \xi_{1 j}^{\left(r_{1}-j\right)}-k_{1 j} \xi_{1 j}^{(j-1)}\right)\right. \\
\dot{\boldsymbol{\theta}}_{f_{i}}=-\gamma_{f_{i}} \boldsymbol{\xi}_{f_{i}}(\mathbf{x}) \mathbf{B}_{i}^{T} \mathbf{P}_{i} \overline{\mathbf{e}}_{i} \quad i=1, \ldots, m \\
\dot{\boldsymbol{\theta}}_{g_{i j}}=-\gamma_{g_{i j}} \boldsymbol{\xi}_{g_{i j}}(\mathbf{x}) \mathbf{B}_{i}^{T} \mathbf{P}_{i} \overline{\mathbf{e}}_{i} u_{j} \quad i, j=1, \ldots, m \\
u_{d i}=\frac{1}{2 \rho_{i}^{2}} \mathbf{B}_{i}^{T} \mathbf{P}_{i} \overline{\mathbf{e}}_{i} \quad i=1, \ldots, m
\end{gathered}
$$

Then the following $H_{\infty}$ tracking performance can be obtained:

$$
\begin{aligned}
\int_{0}^{T} \overline{\mathbf{e}}^{T} \mathbf{Q} \overline{\mathbf{e}} d t \leq & \overline{\mathbf{e}}^{T}(0) \mathbf{P} \overline{\mathbf{e}}(0)+\sum_{i=1}^{m} \frac{1}{\gamma_{f_{i}}} \widetilde{\boldsymbol{\theta}}_{f_{i}}^{T}(0) \widetilde{\boldsymbol{\theta}}_{f_{i}}(0) \\
& +\sum_{i, j=1}^{m} \frac{1}{\gamma_{g_{i j}}} \widetilde{\boldsymbol{\theta}}_{g_{i j}}^{T}(0) \widetilde{\boldsymbol{\theta}}_{g_{i j}}(0)+\sum_{i=1}^{m} \rho_{i}^{2} \int_{0}^{T} \omega_{i}^{2} d t
\end{aligned}
$$

where $\gamma_{f_{i}}(i=1, \ldots, m)$ and $\gamma_{g_{i j}}(i, j=1, \ldots, m)$ are positive adaptive scalar, $\rho_{i}(i=1, \ldots, m)$ are positive parameters representing for prescribed disturbance attenuation levels, $\overline{\mathbf{e}}=$ $\left[\overline{\mathbf{e}}_{1}^{T}, \ldots, \overline{\mathbf{e}}_{m}^{T}\right]^{T}, \mathbf{Q}=\operatorname{diag}\left[\mathbf{Q}_{1}, \ldots, \mathbf{Q}_{m}\right]$, and $\mathbf{Q}_{i} \in \mathbf{R}^{m \times m}(i=$ $1, \ldots, m)$ are arbitrary symmetric positive definite matrices, and $\mathbf{P}=\operatorname{diag}\left[\mathbf{P}_{1}, \ldots, \mathbf{P}_{m}\right]$ and $\mathbf{P}_{i} \in \mathbf{R}^{m \times m}(i=1, \ldots, m)$ are symmetric positive definite solution of the following equations:

$$
\mathbf{P}_{i} \mathbf{A}_{i}+\mathbf{A}_{i}^{T} \mathbf{P}_{i}=-\mathbf{Q}_{i}
$$

Proof. For the $i$ th subsystem of (4), consider the following Lyapunov function candidate:

$$
V_{i}=\frac{1}{2} \overline{\mathbf{e}}_{i}^{T} \mathbf{P}_{i} \overline{\mathbf{e}}_{i}+\frac{1}{2 \gamma_{f_{i}}} \widetilde{\boldsymbol{\theta}}_{f_{i}}^{T} \widetilde{\boldsymbol{\theta}}_{f_{i}}+\frac{1}{2} \sum_{i=1}^{m} \frac{1}{\gamma_{g_{i j}}} \widetilde{\boldsymbol{\theta}}_{g_{i j}^{T}}^{T} \widetilde{\boldsymbol{\theta}}_{g_{i j}} .
$$

Differentiating (57) and considering (49) yield

$$
\begin{aligned}
\dot{V}_{i}= & \frac{1}{2} \dot{\mathbf{e}}_{i}^{T} \mathbf{P}_{i} \overline{\mathbf{e}}_{i}+\frac{1}{2} \overline{\mathbf{e}}_{i}^{T} \mathbf{P}_{i} \dot{\overline{\mathbf{e}}}_{i}+\frac{1}{\gamma_{f_{i}}} \dot{\tilde{\boldsymbol{\theta}}}_{f_{i}}^{T} \widetilde{\boldsymbol{\theta}}_{f_{i}}+\sum_{i=1}^{m} \frac{1}{\gamma_{g_{i j}}} \dot{\tilde{\boldsymbol{\theta}}}_{g_{i j}^{T}} \widetilde{\boldsymbol{\theta}}_{g_{i j}} \\
= & \frac{1}{2} \overline{\mathbf{e}}_{i}^{T}\left(\mathbf{P}_{i} \mathbf{A}_{i}+\mathbf{A}_{i}^{T} \mathbf{P}_{i}\right) \overline{\mathbf{e}}_{i}+\frac{1}{2}\left(-u_{d i}+\omega_{i}\right)\left(\mathbf{B}_{i}^{T} \mathbf{P}_{i} \overline{\mathbf{e}}_{i}+\overline{\mathbf{e}}_{i}^{T} \mathbf{P}_{i} \mathbf{B}_{i}\right) \\
& +\frac{1}{\gamma_{f_{i}}}\left[\gamma_{f_{i}} \overline{\mathbf{e}}_{i}^{T} \mathbf{P}_{i} \mathbf{B}_{i} \xi_{f_{i}}^{T}(\mathbf{x})+\dot{\tilde{\boldsymbol{\theta}}}_{f_{i}}^{T}\right] \widetilde{\boldsymbol{\theta}}_{f_{i}} \\
& +\sum_{i=1}^{m} \frac{1}{\gamma_{g_{i j}}}\left[\gamma_{g_{i j}} \overline{\mathbf{e}}_{i}^{T} \mathbf{P}_{i} \mathbf{B}_{i} \xi_{g_{i j}^{T}}^{T}(\mathbf{x}) u_{j}+\dot{\tilde{\boldsymbol{\theta}}}_{g_{i j}^{T}}\right] \widetilde{\boldsymbol{\theta}}_{g_{i j} .}
\end{aligned}
$$

Substituting (52)-(54) into (58), we obtain

$$
\begin{aligned}
\dot{V}_{i} & =-\frac{1}{2} \overline{\mathbf{e}}_{i}^{T} \mathbf{Q}_{i} \overline{\mathbf{e}}_{i}+\frac{1}{2} \omega_{i}\left(\mathbf{B}_{i}^{T} \mathbf{P}_{i} \overline{\mathbf{e}}_{i}+\overline{\mathbf{e}}_{i}^{T} \mathbf{P}_{i} \mathbf{B}_{i}\right)-\frac{1}{2 \rho_{i}^{2}} \overline{\mathbf{e}}_{i}^{T} \mathbf{P}_{i} \mathbf{B}_{i} \mathbf{B}_{i}^{T} \mathbf{P}_{i} \overline{\mathbf{e}}_{i} \\
& =-\frac{1}{2} \overline{\mathbf{e}}_{i}^{T} \mathbf{Q}_{i} \overline{\mathbf{e}}_{i}+\frac{1}{2} \rho_{i}^{2} \omega_{i}^{2}-\frac{1}{2}\left(\frac{1}{\rho_{i}} \overline{\mathbf{e}}_{i}^{T} \mathbf{P}_{i} \mathbf{B}_{i}-\rho_{i} \omega_{i}\right)^{2} \\
& \leq-\frac{1}{2} \overline{\mathbf{e}}_{i}^{T} \mathbf{Q}_{i} \overline{\mathbf{e}}_{i}+\frac{1}{2} \rho_{i}^{2} \omega_{i}^{2} .
\end{aligned}
$$

Integrating both sides of the above inequality from 0 to $T$ yields

$$
\frac{1}{2} \int_{0}^{T} \overline{\mathbf{e}}_{i}^{T} \mathbf{Q}_{i} \overline{\mathbf{e}}_{i} d t \leq V_{i}(0)-V_{i}(T)+\frac{\rho_{i}^{2}}{2} \int_{0}^{T} \omega_{i}^{2} d t .
$$

Since $V_{i}(T)$ is nonnegative, according to the definition of $V_{i}$, the following inequality is obtained:

$$
\begin{aligned}
\int_{0}^{T} \overline{\mathbf{e}}_{i}^{T} \mathbf{Q}_{i} \overline{\mathbf{e}}_{i} d t \leq & \overline{\mathbf{e}}_{i}^{T}(0) \mathbf{P}_{i} \overline{\mathbf{e}}_{i}(0)+\frac{1}{\gamma_{f_{i}}} \widetilde{\boldsymbol{\theta}}_{f_{i}}^{T}(0) \widetilde{\boldsymbol{\theta}}_{f_{i}}(0) \\
& +\sum_{i=1}^{m} \frac{1}{\gamma_{g_{i j}}} \widetilde{\boldsymbol{\theta}}_{g_{i j}}^{T}(0) \widetilde{\boldsymbol{\theta}}_{g_{i j}}(0)+\rho_{i}^{2} \int_{0}^{T} \omega_{i}^{2} d t .
\end{aligned}
$$

Considering the Lyapunov function $V=\sum_{i=1}^{m} V_{i}$, it is easy to obtain the $H_{\infty}$ tracking performance index (55). This completes the proof.

From the above analysis, it is concluded that, in the case of no control saturations, the signals $\xi_{i j}(i=1, \ldots, m ; j=$ $1, \ldots, r_{i}$ ) remain zeros and the control law becomes the same as the standard robust adaptive control law described in the previous section. In the presence of control saturations, $\xi_{i j}$ is nonzero, thus giving rise to a modified tracking error $\bar{e}_{i}=y_{d i}-y_{i}-\xi_{i 1}$, which is used in fuzzy parameter update laws. The auxiliary control term $\mathbf{u}_{a w}$ in (40) will be used to compensate the effects of control saturations. Note that the fuzzy parameter update laws (52) and (53) are similar to the corresponding update laws (30) and (31) derived in the standard fuzzy approximation based control problem with tracking error $e_{i}$ being replaced by the modified tracking error $\bar{e}_{i}$. The use of the modified tracking error in the fuzzy update laws is crucial in preventing actuator constraints in online approximation schemes. 
Corollary 7. For the ith subsystem of (4), it is assumed that $\int_{0}^{T} d_{i}^{2} d t<\infty$. If the control law (40), the auxiliary control term (51), and the parameter update laws (52)-(54) are adopted, then the following statements hold.

(i) The closed loop system is stable, and the signals $\overline{\mathbf{e}}_{i}, \boldsymbol{\theta}_{f_{i}}$, $\boldsymbol{\theta}_{g_{i j}}$, and $u_{i}$ are bounded.

(ii) The steady modified tracking error satisfies $\lim _{t \rightarrow \infty} \bar{e}_{i}=$ 0 , and the bound of the transient modified tracking error will be given as follows:

$$
\begin{aligned}
& \left\|\bar{e}_{i}\right\|^{2} \\
& \leq \frac{2\left[\left(1 / \gamma_{f_{i}}\right) \tilde{\boldsymbol{\theta}}_{f_{i}}^{T}(\mathbf{0}) \widetilde{\boldsymbol{\theta}}_{f_{i}}(\mathbf{0})+\sum_{i=1}^{m}\left(1 / \gamma_{g_{i j}}\right) \tilde{\boldsymbol{\theta}}_{g_{i j}}^{T}(\mathbf{0}) \tilde{\boldsymbol{\theta}}_{g_{i j}}(\mathbf{0})\right]}{\lambda_{\min }\left(\mathbf{Q}_{i}\right)} \\
& \quad+\frac{2 \overline{\mathbf{e}}_{i}^{T}(\mathbf{0}) \mathbf{P}_{i} \overline{\mathbf{e}}_{i}(\mathbf{0})+\rho_{i}^{2} \int_{0}^{T} \omega_{i}^{2} d t}{\lambda_{\min }\left(\mathbf{Q}_{i}\right)} .
\end{aligned}
$$

Proof. From (59), it can be obtained that

$$
\dot{V}_{i} \leq-c_{i} V_{i}+\mu_{i}
$$

where $c_{i}=\min \left\{\lambda_{v}, 1 / \gamma_{f_{i}}, 1 / \gamma_{g_{i j}}\right\}, \lambda_{v}=\min \left\{\inf \lambda_{\min }\left(\mathbf{Q}_{i}\right) /\right.$ $\left.\sup \lambda_{\max }\left(\mathbf{Q}_{i}\right)\right\}, M_{i}=\max \left(\boldsymbol{\theta}_{f_{i}}\right), M_{i j}=\max \left(\boldsymbol{\theta}_{g_{i j}}\right), \mu_{i}=$ $M_{i}^{2} / 2 \gamma_{f_{i}}+\sum_{j=1}^{m}\left(1 / 2 \gamma_{g_{i j}}\right) M_{i j}^{2}+(1 / 2) \rho_{i}^{2} \bar{\omega}_{i}^{2}, \bar{\omega}_{i}=\sup \left\|\omega_{i}\right\|$, and $\lambda_{\min }\left(\mathbf{Q}_{i}\right)$ and $\lambda_{\max }\left(\mathbf{Q}_{i}\right)$ are the minimum and maximum eigenvalue of $\mathbf{Q}_{i}$, respectively.

From inequality (63), all the signals $\overline{\mathbf{e}}_{i}, \boldsymbol{\theta}_{f_{i}}, \boldsymbol{\theta}_{g_{i j}}$, and $u_{i}$ are bounded by using Barbalat's lemma [8].

On the other hand, from (59), it can be obtained that

$$
\dot{V}_{i} \leq-\frac{1}{2} \lambda_{\min }\left(\mathbf{Q}_{i}\right)\left\|\overline{\mathbf{e}}_{i}\right\|^{2}+\frac{1}{2} \rho_{i}^{2} \omega_{i}^{2} .
$$

From the above inequality, one obtains

$$
\left\|\bar{e}_{i}\right\|^{2} \leq\left\|\overline{\mathbf{e}}_{i}\right\|^{2} \leq \frac{-2 \dot{V}_{i}+\rho_{i}^{2} \omega_{i}^{2}}{\lambda_{\min }(Q)}
$$

Hence,

$$
\begin{aligned}
\left\|\bar{e}_{i}\right\|^{2} & =\int_{0}^{T} \bar{e}_{i}^{2} d t \leq \frac{2\left[V_{i}(0)-V_{i}(T)\right]+\rho_{i}^{2} \int_{0}^{T} \omega_{i}^{2} d t}{\lambda_{\min }\left(\mathbf{Q}_{i}\right)} \\
& \leq \frac{2 V_{i}(0)+\rho_{i}^{2} \int_{0}^{T} \omega_{i}^{2} d t}{\lambda_{\min }\left(\mathbf{Q}_{i}\right)} .
\end{aligned}
$$

According to the definition of $V_{i},(62)$ is obtained. Assumption 4 implies $\int_{0}^{T} \omega_{i}^{\prime 2} d t<\infty$; then $\int_{0}^{T} \omega_{i}^{2} d t=$ $\int_{0}^{T}\left(\omega_{i}^{\prime}-d_{i}\right)^{2} d t<\infty$. Therefore, $\omega_{i} \in L_{2}$. From (66) we can conclude that $\bar{e}_{i} \in L_{2}$. Using Barbalat's lemma, it follows that $\lim _{t \rightarrow \infty} \bar{e}_{i}(t)=0$. This completes the proof.
Remark 8. According to Theorem 6, the ith subsystem of (4) achieves a $H^{\infty}$ tracking performance with a prescribed disturbance attenuation level $\rho_{i}$; that is, the $L_{2}$ gain from $\omega_{i}$ to the modified tracking error $\bar{e}_{i}$ is equal or less than $\rho_{i}$.

Remark 9. As indicated from Corollary 7, the steady modified tracking error $\bar{e}_{i}$ will converge to zero. The bound of the transient modified tracking error $\bar{e}_{i}$ is an explicit function of the design parameters and the external disturbance and fuzzy approximation error $\omega_{i}$. The bound can be decreased by choosing the initial estimates $\boldsymbol{\theta}_{f_{i}}(\mathbf{0}), \boldsymbol{\theta}_{g_{i j}}(\mathbf{0})$ closing to the true values $\boldsymbol{\theta}_{f_{i}}^{*}, \boldsymbol{\theta}_{g_{i j}}^{*}$. The effects of parameter initial estimate errors on the transient tracking performance can be reduced by increasing the adaptive gain values $\gamma_{f_{i}}, \gamma_{g_{i j}}$, and $\lambda_{\min }\left(\mathbf{Q}_{i}\right)$. Furthermore, the effect of external disturbance and fuzzy approximation error $\omega_{i}$ on the transient tracking performance can be reduced by decreasing $\rho_{i}$ and increasing $\lambda_{\text {min }}\left(\mathbf{Q}_{i}\right)$. Small $\rho_{i}$ implies high disturbance attenuation level.

\section{Simulation Examples}

The attitude tracking control problem of a rigid body satellite system is simulated in this section to illustrate the effectiveness of the robust adaptive fuzzy controllers proposed in this paper. The mathematical model of the satellite attitude system can be reformulated to the general form of uncertain nonlinear MIMO system as follows $[42,44]$ :

$$
\ddot{\mathbf{y}}=\mathbf{F}(\mathbf{x})+\mathbf{G}(\mathbf{x}) \mathbf{u}+\mathbf{d},
$$

where $\mathbf{x}=[\mathbf{q}, \boldsymbol{\omega}]^{T}$ is the state vector, where $\mathbf{q}=$ $\left[q_{0}, q_{1}, q_{2}, q_{3}\right]^{T}$ is the attitude quaternion in the body-fixed reference frame relative to the inertial frame satisfying $q_{0}^{2}+$ $q_{1}^{2}+q_{2}^{2}+q_{3}^{2}=1$, here $q_{0}$ is chosen as $q_{0}=\sqrt{1-q_{1}^{2}-q_{2}^{2}-q_{3}^{2}}$; $\boldsymbol{\omega}=\left[\omega_{x}, \omega_{y}, \omega_{z}\right]^{T}$ is the angular velocity of the body-fixed reference relative to the inertial frame. $y=\left[q_{1}, q_{2}, q_{3}\right]^{T}$ is the output vector, $\mathbf{u}=\left[u_{1}, u_{2}, u_{3}\right]^{T}$ is the control torque vector, $\mathbf{d}^{\prime} \in \mathbf{R}^{3}$ denotes the bounded external disturbance torques, and $\mathbf{d} \triangleq \mathbf{G}(\mathbf{x}) \mathbf{d}^{\prime} . \mathbf{F}(\mathbf{x})$ and $\mathbf{G}(\mathbf{x})$ are expressed as follows:

$$
\begin{aligned}
f_{1}(\mathbf{x})= & -\frac{1}{4} q_{1}\left(\omega_{x}^{2}+\omega_{y}^{2}+\omega_{z}^{2}\right)+\frac{I_{x}-I_{y}}{2 I_{z}} q_{2} \omega_{x} \omega_{y} \\
& +\frac{I_{x}-I_{z}}{2 I_{y}} q_{3} \omega_{x} \omega_{z}+\frac{I_{y}-I_{z}}{2 I_{x}} q_{0} \omega_{y} \omega_{z} \\
f_{2}(\mathbf{x})= & -\frac{1}{4} q_{2}\left(\omega_{x}^{2}+\omega_{y}^{2}+\omega_{z}^{2}\right)+\frac{I_{y}-I_{x}}{2 I_{z}} q_{1} \omega_{x} \omega_{y} \\
& +\frac{I_{z}-I_{x}}{2 I_{y}} q_{0} \omega_{x} \omega_{z}+\frac{I_{y}-I_{z}}{2 I_{x}} q_{3} \omega_{y} \omega_{z}
\end{aligned}
$$




$$
\begin{aligned}
& f_{3}(\mathbf{x})=-\frac{1}{4} q_{3}\left(\omega_{x}^{2}+\omega_{y}^{2}+\omega_{z}^{2}\right)+\frac{I_{x}-I_{y}}{2 I_{z}} q_{0} \omega_{x} \omega_{y} \\
&+\frac{I_{z}-I_{x}}{2 I_{y}} q_{1} \omega_{x} \omega_{z}+\frac{I_{z}-I_{y}}{2 I_{x}} q_{2} \omega_{y} \omega_{z} \\
& \mathbf{G}(\mathbf{x})=\left[\begin{array}{ccc}
\frac{q_{0}}{2 I_{x}} & -\frac{q_{3}}{2 I_{y}} & \frac{q_{2}}{2 I_{z}} \\
\frac{q_{3}}{2 I_{x}} & \frac{q_{0}}{2 I_{y}} & -\frac{q_{1}}{2 I_{z}} \\
-\frac{q_{2}}{2 I_{x}} & \frac{q_{1}}{2 I_{y}} & \frac{q_{0}}{2 I_{z}}
\end{array}\right]
\end{aligned}
$$

where $I_{x}, I_{y}$, and $I_{z}$ are the principal central moments of inertia of the satellite.

Within this simulation, the nonlinear functions $\mathbf{F}(\mathbf{x})$ and $\mathbf{G}(\mathbf{x})$ are assumed completely unknown, that is, the fuzzy adaptive controllers do not require the knowledge of the system's model. In fact, the dynamic model of the satellite attitude system is only required for simulation purpose.

Since the components of $\mathbf{F}(\mathbf{x})$ and $\mathbf{G}(\mathbf{x})$ are assumed unknown, three fuzzy logic systems in the form of (12) are used to approximate the elements of $\mathbf{F}(\mathbf{x})$, and nine are used to approximate the elements of $\mathbf{G}(\mathbf{x})$. The fuzzy logic systems used to describe $\mathbf{F}(\mathbf{x})$ have $q_{1}, q_{2}, q_{3}, \omega_{1}, \omega_{2}$, and $\omega_{3}$ as inputs, and the ones used to describe $\mathbf{G}(\mathbf{x})$ have $q_{1}, q_{2}$, and $q_{3}$ as inputs. For each state variable $\mathbf{x}=\left[q_{1}, q_{2}, q_{3}, \omega_{1}, \omega_{2}, \omega_{3}\right]^{T}$, we define seven Gaussian membership functions as

$$
\begin{aligned}
& \mu_{F_{i}^{1}}\left(x_{i}\right)=\frac{1}{1+\exp \left(-5\left(x_{i}+0.6\right)\right)}, \\
& \mu_{F_{i}^{2}}\left(x_{i}\right)=\exp \left(-0.5\left(x_{i}+0.4\right)^{2}\right) \\
& \mu_{F_{i}^{3}}\left(x_{i}\right)=\exp \left(-0.5\left(x_{i}+0.2\right)^{2}\right), \\
& \mu_{F_{i}^{4}}\left(x_{i}\right)=\exp \left(-0.5 x_{i}^{2}\right) \\
& \mu_{F_{i}^{5}}\left(x_{i}\right)=\exp \left(-0.5\left(x_{i}-0.2\right)^{2}\right), \\
& \mu_{F_{i}^{6}}\left(x_{i}\right)=\exp \left(-0.5\left(x_{i}-0.4\right)^{2}\right) \\
& \mu_{F_{i}^{7}}\left(x_{i}\right)=\frac{1}{1+\exp \left(-5\left(x_{i}-0.6\right)\right)}, \\
& i=1,2,3,4,5,6 .
\end{aligned}
$$

The fuzzy basis function vectors are chosen as follows:

$$
\begin{array}{r}
\xi_{f_{i}}=\left[\frac{\prod_{i=1}^{6} \mu_{F_{i}^{1}}\left(x_{i}\right)}{\sum_{j=1}^{7} \prod_{i=1}^{6} \mu_{F_{i}^{j}}\left(x_{i}\right)}, \ldots, \frac{\prod_{i=1}^{6} \mu_{F_{i}^{7}}\left(x_{i}\right)}{\sum_{j=1}^{7} \prod_{i=1}^{6} \mu_{F_{i}^{j}}\left(x_{i}\right)}\right]^{T} \\
i=1,2,3
\end{array}
$$

$$
\begin{array}{r}
\xi_{g_{i j}}=\left[\frac{\prod_{i=1}^{3} \mu_{F_{i}^{1}}\left(x_{i}\right)}{\sum_{j=1}^{7} \prod_{i=1}^{3} \mu_{F_{i}^{j}}\left(x_{i}\right)}, \ldots, \frac{\prod_{i=1}^{3} \mu_{F_{i}^{7}}\left(x_{i}\right)}{\sum_{j=1}^{7} \prod_{i=1}^{3} \mu_{F_{i}^{j}}\left(x_{i}\right)}\right]^{T} \\
i, j=1,2,3 .
\end{array}
$$

Then we obtain fuzzy logic systems

$$
\begin{gathered}
\widehat{f}_{i}\left(\mathbf{x} \mid \boldsymbol{\theta}_{f_{i}}\right)=\boldsymbol{\theta}_{f_{i}}^{T} \boldsymbol{\xi}_{f_{i}}(\mathbf{x}) \quad i=1,2,3 \\
\widehat{g}_{i j}\left(\mathbf{x} \mid \boldsymbol{\theta}_{g_{i j}}\right)=\boldsymbol{\theta}_{g_{i j}^{T}}^{T} \xi_{g_{i j}}(\mathbf{x}) \quad i, j=1,2,3 .
\end{gathered}
$$

Using (71) approximate the unknown functions $f_{i}$ and $g_{i j}$, respectively.

For the given coefficients $k_{i 1}=0.25(i=1,2,3)$ and $k_{i 2}=$ $0.5(i=1,2,3)$, we have

$$
\mathbf{A}_{1}=\mathbf{A}_{2}=\mathbf{A}_{3}=\left[\begin{array}{cc}
0 & 1 \\
-0.25 & -0.5
\end{array}\right] \quad \mathbf{B}_{1}=\mathbf{B}_{2}=\mathbf{B}_{3}=\left[\begin{array}{l}
0 \\
1
\end{array}\right] .
$$

Selecting $\mathbf{Q}_{i}=\operatorname{diag}[0.5,0.5]$ and solving (34), we obtain

$$
\mathbf{P}_{i}=\left[\begin{array}{cc}
1.625 & 0.5 \\
0.5 & 3
\end{array}\right] \quad i=1,2,3 .
$$

The parameters of the adaptive fuzzy controllers are chosen as $\gamma_{f_{i}}=1 \times 10^{-3}, \gamma_{g_{i j}}=1 \times 10^{-3}$, and $\rho_{i}=0.5$, $i, j=1,2,3$.

The initial attitude quaternion is $\mathbf{q}(0)=[0.9014,0.25$, $0.25,0.25]^{T}$, and the initial value of the angular velocity is $\boldsymbol{\omega}(0)=\left[\begin{array}{lll}0.005 & 0.005 & 0.005\end{array}\right]^{T} \mathrm{rad} / \mathrm{s}$. The parameters of system are given as $I_{x}=187.5 \mathrm{~kg} \cdot \mathrm{m}^{2}, I_{y}=468.5 \mathrm{~kg} \cdot \mathrm{m}^{2}$, and $I_{z}=468.5 \mathrm{~kg} \cdot \mathrm{m}^{2}$. The external disturbance torque vector is given by $\mathbf{d}^{\prime}=[0.3 \sin (0.05 t), 0.3 \cos (0.05 t)$, $-0.3 \sin (0.05 t)]^{T} \mathrm{~N} \cdot \mathrm{m}$. The initial values of the parameters $\boldsymbol{\theta}_{f_{i}}$ and $\boldsymbol{\theta}_{g_{i j}}$ are set to random values uniformly distributed between $[0,1]$. The desired output trajectory is chosen as $\mathbf{y}_{d}=[0.5 \sin (0.005 t), 0.5 \sin (0.005 t), 0.5 \sin (0.005 t)]^{T}$. The control objective is to force the system output $\mathbf{y}$ to track the desired output trajectory $\mathbf{y}_{d}$.

4.1. Without Input Saturation. In this section, the tracking control problem is simulated to demonstrate the effectiveness of robust adaptive fuzzy controller (24) proposed in Section 3.1.

Using the control law (24) and (30)-(32), simulation results are presented in Figures 1 and 2. Figure 1 shows the curves of the system outputs and its reference trajectories, which indicates that the robust adaptive fuzzy controller achieves a good performance in tracking control problem and the effects of fuzzy approximation errors and external disturbances on tracking errors are effectively attenuated. Figure 2 shows that the control inputs can be carried out feasibly without any constraints. The control signals are obtained by certainty equivalence principle. Therefore, they do not satisfy the control input limitations naturally. 

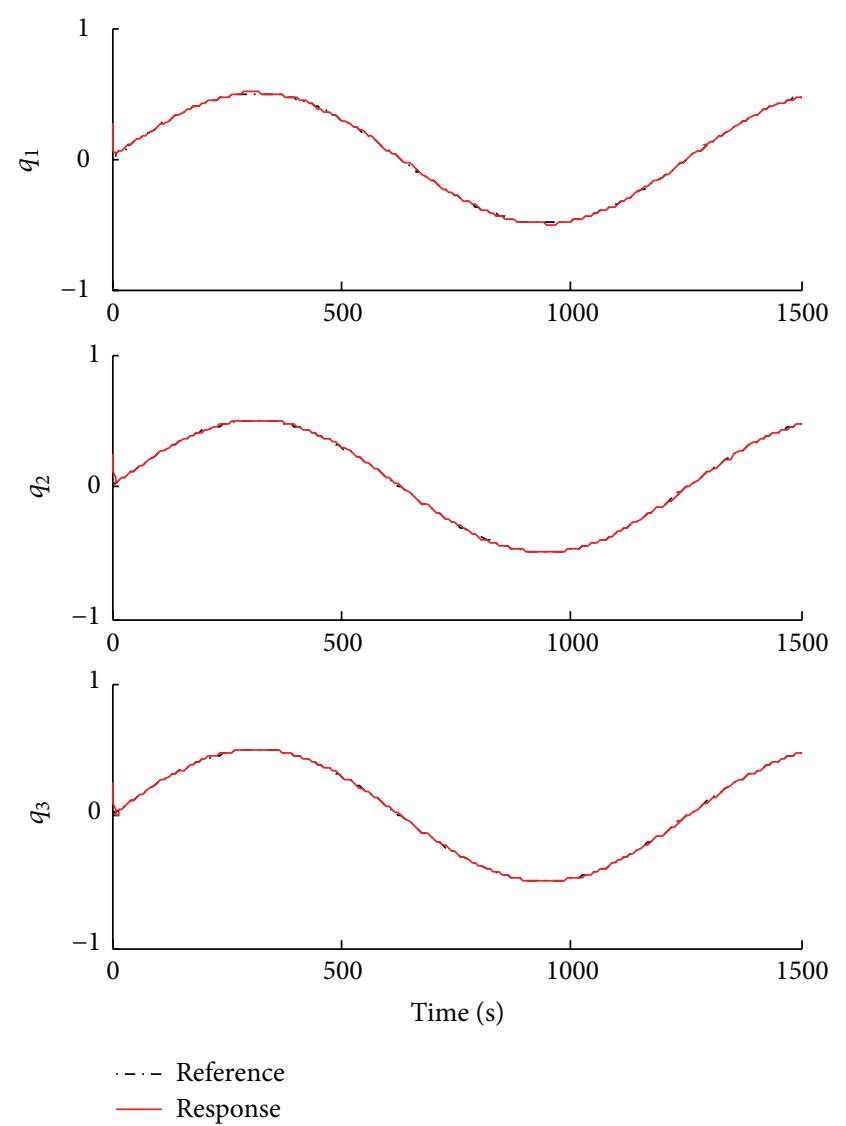

Figure 1: Trajectories of outputs without saturation.

4.2. Actuator Amplitude Saturation. In order to demonstrate that the proposed adaptive control scheme (40) can work effectively under actuator amplitude saturation, numerical simulations have been performed and presented in this section.

Consider satellite attitude model (67) with the same disturbances and system initial conditions mentioned above. The control input vector $\mathbf{u}=\left[u_{1}, u_{2}, u_{3}\right]^{T}$ has amplitude limits $\left|u_{i}\right| \leq 1 \mathrm{~N} \cdot \mathrm{m}, i=1,2,3$. The auxiliary system is constructed as follows:

$$
\begin{aligned}
& \dot{\xi}_{i 1}=\xi_{i 2}-\lambda_{i 1} \xi_{i 1} \\
& \dot{\xi}_{i 2}=-\lambda_{i 2} \xi_{i 2}+\sum_{j=1}^{3} \widehat{g}_{i j}\left(\mathbf{x} \mid \boldsymbol{\theta}_{g_{i j}}\right)\left(u_{j}-u_{c j}\right) \quad i=1,2,3,
\end{aligned}
$$

where $\lambda_{i j}=1(i=1,2,3 ; j=1,2)$ and $\xi_{i j}(0)=0(i=$ $1,2,3 ; j=1,2)$.

Using the control law (40), the auxiliary control term (51), the parameters update laws (52)-(53), and the robust compensation term (54), we can get the simulations in Figures 3 and 4.

The system outputs and its reference trajectories are shown in Figure 3, which indicate that the outputs track their reference trajectories well in spite of the external disturbances, system uncertainties, and actuator amplitude saturation. Figure 4 shows the trajectories of the control
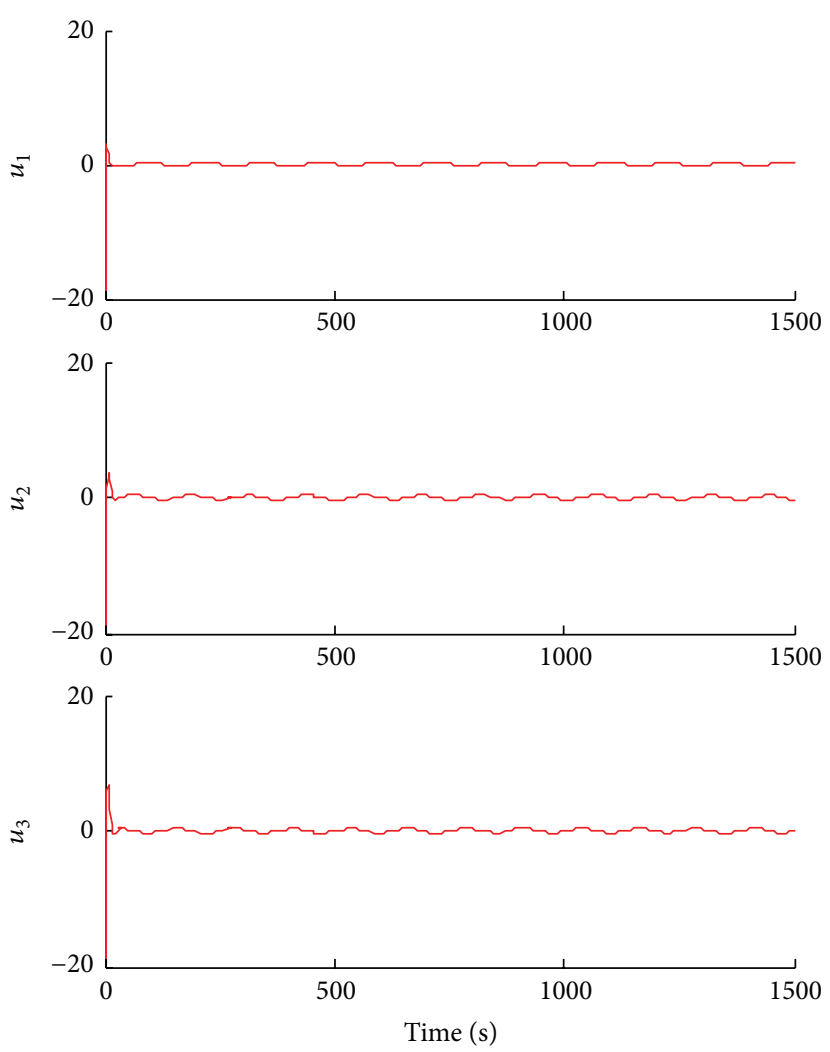

FIgURE 2: Trajectories of control inputs without saturation.
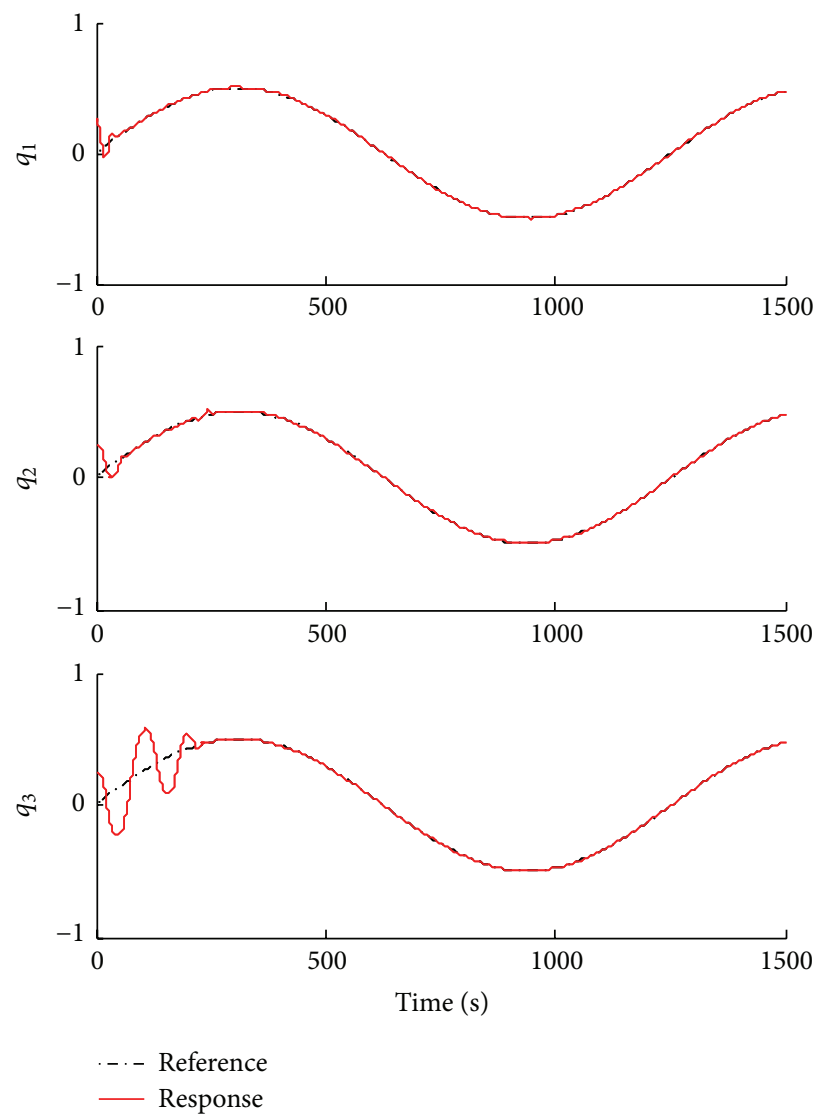

FIgURE 3: Trajectories of outputs with amplitude saturation. 

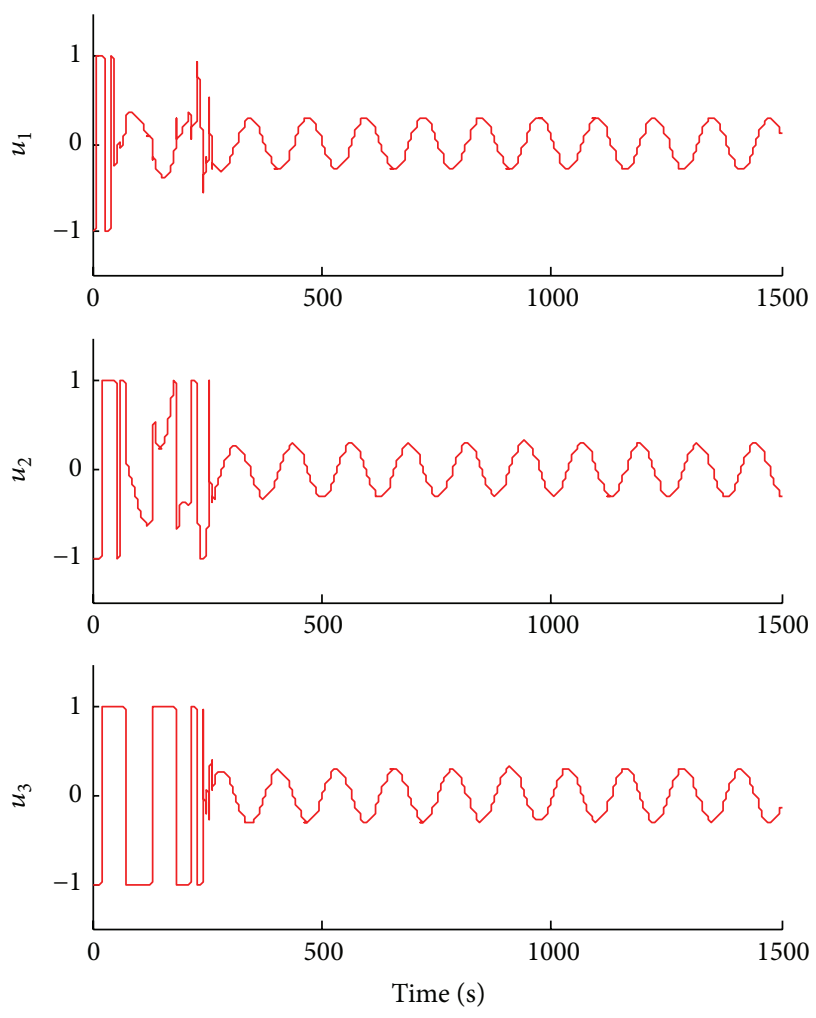

FIGURE 4: Trajectories of control inputs with amplitude saturation.

inputs with actuator amplitude saturation. From these simulations, it is obvious that proposed control scheme (40) can achieve a good tracking performance when the actuator amplitude limits are considered.

4.3. Actuator Amplitude and Rate Saturation. For further analysis, actuator amplitude and rate saturations are considered. The control input vector $\mathbf{u}=\left[u_{1}, u_{2}, u_{3}\right]^{T}$ has the amplitude and rate limitation $\left|u_{i}\right| \leq 1,\left|\dot{u}_{i}\right| \leq 2, i=1,2,3$. The other initial parameters are the same as mentioned in Section 4.2.

According to (41), the dynamics of control inputs are expressed as follows:

$$
\dot{u}_{i}=S_{1}\left(\omega_{i} S_{1}\left(u_{c i}\right)-u_{i}\right) \quad i=1,2,3,
$$

where $\omega_{i}=20.5(i=1,2,3)$.

Using control law (40), the actuator amplitude and rate constraints (41), the auxiliary control term (51), the parameters update laws (52)-(53), and the robust compensation term (54), simulation results are presented in Figures 5-8. Figure 5 shows the curves of outputs and their reference trajectories, which indicate that a good tracking control performance is still achieved under actuator amplitude and rate constraint conditions. The control signals and their derivatives are given in Figures 6 and 7. It is observed that the control signals satisfy the amplitude and rate limitations. That is, the proposed robust control scheme for the satellite attitude control system can prevent the control signals from reaching amplitude and
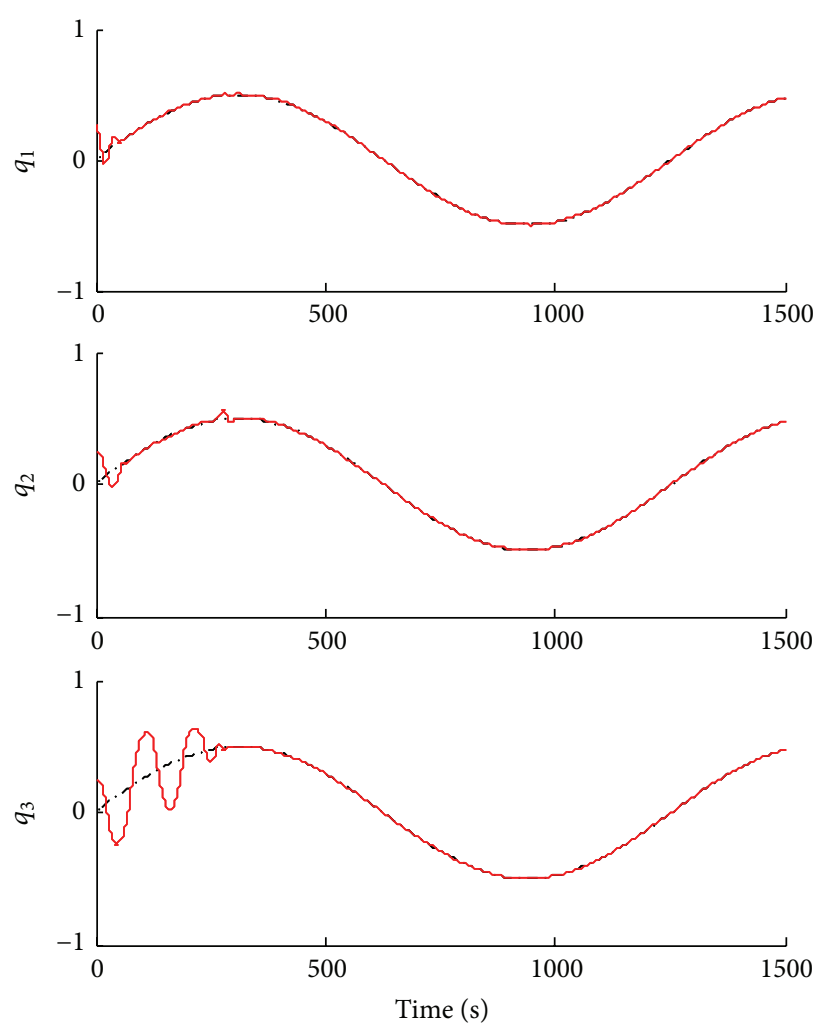

-..- Reference

- Response

FIGURE 5: Trajectories of outputs with amplitude and rate saturation.
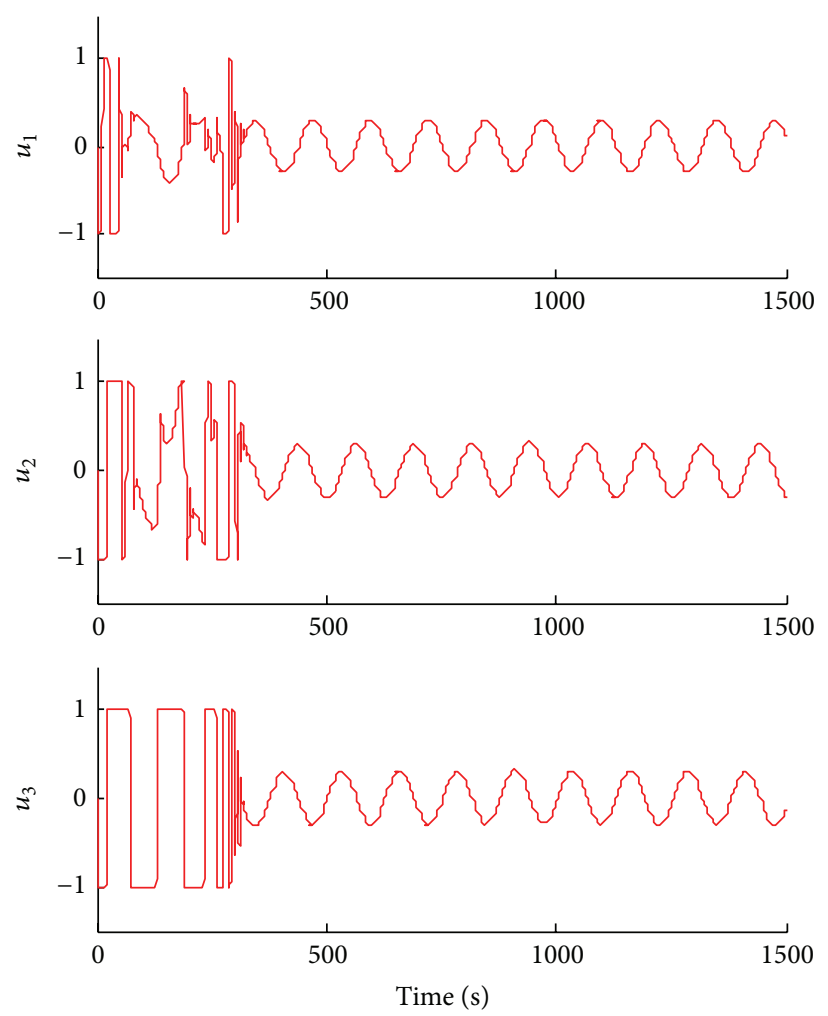

FIGURE 6: Trajectories of control inputs with amplitude and rate saturation. 

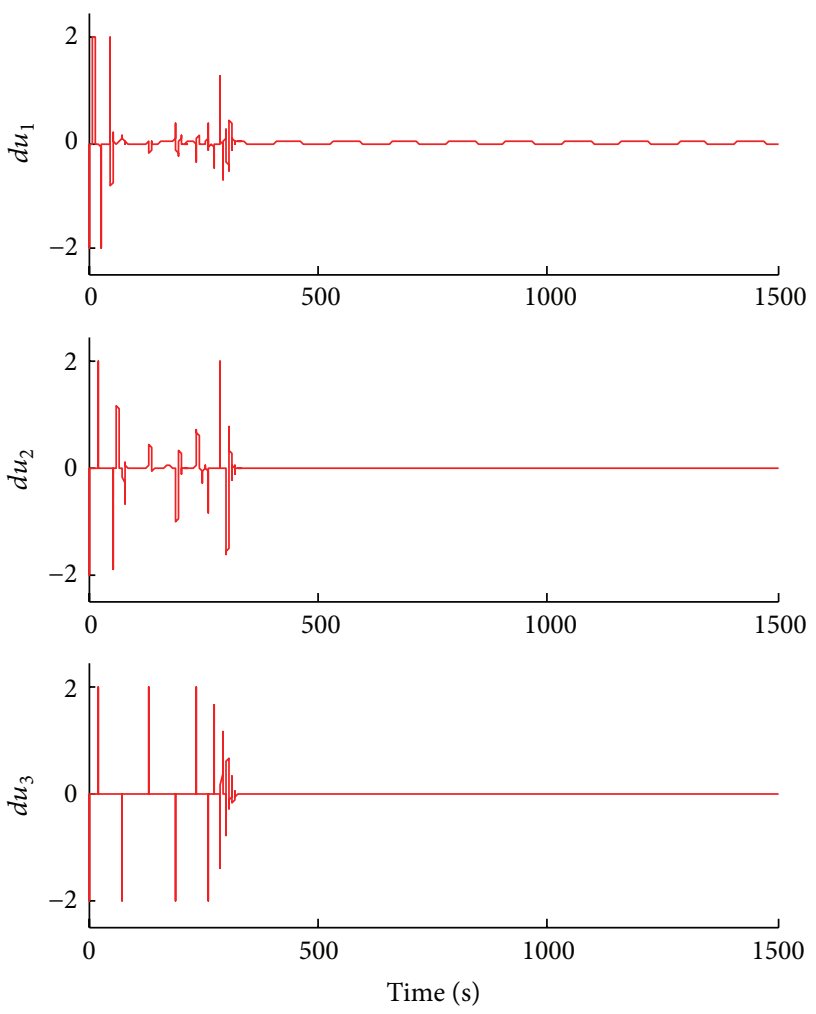

FIGURE 7: Derivatives of control inputs.
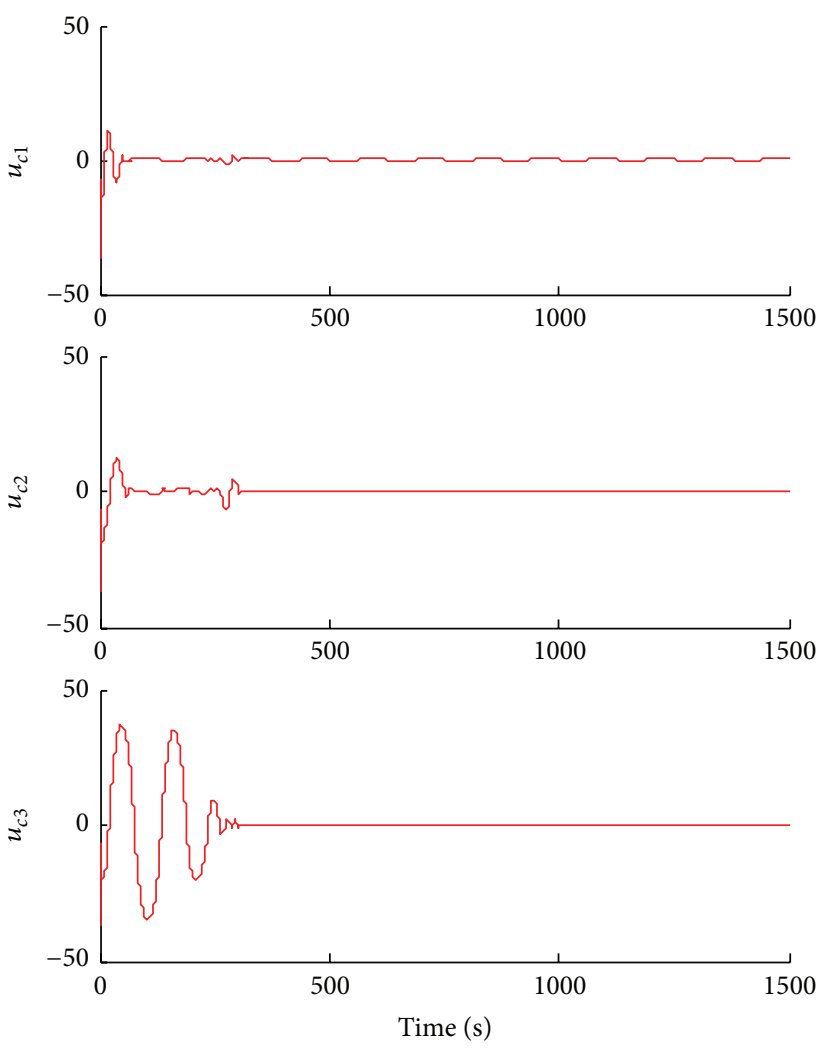

FIGURE $8: u_{c 1}, u_{c 2}$, and $u_{c 3}$. rate saturation limits. Figure 8 shows the signals $u_{c i}(i=$ $1,2,3)$. Obviously, they do not satisfy the control input limitations.

From the aforementioned simulations, it is demonstrated that the robust adaptive fuzzy tracking controller proposed in this paper not only can generate control inputs that satisfy actuator amplitude and rate saturations but also can effectively attenuate the effects of approximation error and extern disturbance on tracking errors. Thus, the proposed robust adaptive control scheme is valid for satellite attitude control system with actuator amplitude and rate saturation.

\section{Conclusions}

In this work, a robust fuzzy tracking control approach has been presented for a class of uncertain nonlinear MIMO systems in the presence of input saturation and external disturbances. Fuzzy logic systems are used to approximate the unknown system nonlinear terms. An auxiliary system is constructed to compensate the effects of actuator saturations, and then the actuator saturations can be augmented into the controller. The modified tracking error is introduced and used in fuzzy parameter update laws. A robust compensation control is designed to attenuate the effects of external disturbances and fuzzy approximation errors. The stability properties and tracking performance of the closed-loop system are obtained through Lyapunov analysis. Steady and transient modified tracking errors are analyzed and the bound of modified tracking errors can be adjusted by tuning certain design parameters. The proposed control scheme is applicable to uncertain nonlinear systems not only with actuator amplitude saturation, but also with actuator amplitude and rate saturation. The simulation results of satellite attitude control system are presented to demonstrate the effectiveness of proposed controller. In this paper, the control system relies on the output derivatives, up to $r_{i}-1$ order as in (22), which is also practically restrictive for high order systems. Future research will be concentrated on an observer-based robust adaptive fuzzy control of uncertain nonlinear systems with actuator saturations based on the results of [18-20] and this paper.

\section{Conflict of Interests}

The authors declare that there is no conflict of interests regarding the publication of this paper.

\section{Acknowledgment}

This research is supported by the Fund of Innovation by Graduate School of National University of Defense Technology under Grant B140106.

\section{References}

[1] L.-X. Wang, "Automatic design of fuzzy controllers," Automatica, vol. 35, no. 8, pp. 1471-1475, 1999.

[2] S. C. Tong, J. T. Tang, and T. Wang, "Fuzzy adaptive control of multivariable nonlinear systems," Fuzzy Sets and Systems, vol. 111, no. 2, pp. 153-167, 2000. 
[3] B.-S. Chen, C.-H. Lee, and Y.-C. Chang, " $H_{\infty}$ tracking design of uncertain nonlinear SISO systems: adaptive fuzzy approach," IEEE Transactions on Fuzzy Systems, vol. 4, no. 1, pp. 32-43, 1996.

[4] T. Zhang, S. S. Ge, and C. C. Hang, "Adaptive neural network control for strict-feedback nonlinear systems using backstepping design," Automatica, vol. 36, no. 12, pp. 1835-1846, 2000.

[5] S.-C. Tong, X.-L. He, and H.-G. Zhang, "A combined backstepping and small-gain approach to robust adaptive fuzzy output feedback control," IEEE Transactions on Fuzzy Systems, vol. 17, no. 5, pp. 1059-1069, 2009.

[6] Y. Pan, Y. Zhou, T. Sun, and M. J. Er, "Composite adaptive fuzzy $H_{\infty}$ tracking control of uncertain nonlinear systems," Neurocomputing, vol. 99, pp. 15-24, 2013.

[7] Y.-T. Kim and Z. Z. Bien, "Robust adaptive fuzzy control in the presence of external disturbance and approximation error," Fuzzy Sets and Systems, vol. 148, no. 3, pp. 377-393, 2004.

[8] S. C. Tong and H.-X. Li, "Fuzzy adaptive sliding-mode control for MIMO nonlinear systems," IEEE Transactions on Fuzzy Systems, vol. 11, no. 3, pp. 354-360, 2003.

[9] Y.-C. Chang, "Robust tracking control for nonlinear MIMO systems via fuzzy approaches," Automatica, vol. 36, no. 10, pp. 1535-1545, 2000.

[10] Y.-J. Liu, C. L. P. Chen, G.-X. Wen, and S. Tong, "Adaptive neural output feedback tracking control for a class of uncertain discrete-time nonlinear systems," IEEE Transactions on Neural Networks, vol. 22, no. 7, pp. 1162-1167, 2011.

[11] S. C. Tong, B. Chen, and Y. F. Wang, "Fuzzy adaptive output feedback control for MIMO nonlinear systems," Fuzzy Sets and Systems, vol. 156, no. 2, pp. 285-299, 2005.

[12] Y.-J. Liu, S.-C. Tong, and W. Wang, "Adaptive fuzzy output tracking control for a class of uncertain nonlinear systems," Fuzzy Sets and Systems, vol. 160, no. 19, pp. 2727-2754, 2009.

[13] Y.-J. Liu, W. Wang, S.-C. Tong, and Y.-S. Liu, "Robust adaptive tracking control for nonlinear systems based on bounds of fuzzy approximation parameters," IEEE Transactions on Systems, Man, and Cybernetics Part A: Systems and Humans, vol. 40, no. 1, pp. 170-184, 2010.

[14] Y. N. Yang, J. Wu, and W. Zheng, "Adaptive fuzzy sliding mode control for robotic airship with model uncertainty and external disturbance," Journal of Systems Engineering and Electronics, vol. 23, no. 2, pp. 250-255, 2012.

[15] C. L. P. Chen, Y.-J. Liu, and G.-X. Wen, "Fuzzy neural networkbased adaptive control for a class of uncertain nonlinear stochastic systems," IEEE Transactions on Cybernetics, vol. 44, no. 5, pp. 583-593, 2014.

[16] C. L. P. Chen, G.-X. Wen, Y.-J. Liu, and F.-Y. Wang, "Adaptive consensus control for a class of nonlinear multiagent time-delay systems using neural networks," IEEE Transactions on Neural Networks and Learning Systems, vol. 25, no. 6, pp. 1217-1226, 2014.

[17] Y. J. Liu and S. C. Tong, "Adaptive neural network tracking control of uncertain nonlinear discrete-time systems with nonaffine dead-zone input," IEEE Transactions on Cybernetics, vol. 45, no. 3, pp. 497-505, 2015.

[18] Y.-J. Liu, S. Tong, and C. L. P. Chen, "Adaptive fuzzy control via observer design for uncertain nonlinear systems with unmodeled dynamics," IEEE Transactions on Fuzzy Systems, vol. 21, no. 2, pp. 275-288, 2013.

[19] S. C. Tong, Y. Li, Y. M. Li, and Y. J. Liu, "Observer-based adaptive fuzzy backstepping control for a class of stochastic nonlinear strict-feedback systems," IEEE Transactions on Systems, Man, and Cybernetics-Part B: Cybernetics, vol. 41, no. 6, pp. 16931704, 2011.

[20] S. C. Tong, B. Y. Huo, and Y. M. Li, "Observer-based adaptive decentralized fuzzy fault-tolerant control of nonlinear largescale systems with actuator failures," IEEE Transactions on Fuzzy Systems, vol. 22, no. 1, pp. 1-15, 2014.

[21] J. Farrell, M. Polycarpou, and M. Sharma, Adaptive Backstepping with Magnitude, Rate, and Bandwidth Constraints: Aircraft Longitude Control, California University Riverside, Department of Electrical Engineering, 2006.

[22] X. P. Shi, G. P. Yuan, and L. Li, "Adaptive fuzzy attitude tracking control of spacecraft with input magnitude and rate constraints," in Proceedings of the 31st Chinese Control Conference (CCC '12), pp. 842-846, July 2012.

[23] A. Leonessa, W. M. Haddad, T. Hayakawa, and Y. Morel, "Adaptive control for nonlinear uncertain systems with actuator amplitude and rate saturation constraints," International Journal of Adaptive Control and Signal Processing, vol. 23, no. 1, pp. 7396, 2009.

[24] J. A. Farrell, M. Sharma, and M. Polycarpou, "Longitudinal flight-path control using online function approximation," Journal of Guidance, Control, and Dynamics, vol. 26, no. 6, pp. 885897, 2003.

[25] J. Farrell, M. Sharma, and M. Polycarpou, "Backstepping-based flight control with adaptive function approximation," Journal of Guidance, Control, and Dynamics, vol. 28, no. 6, pp. 1089-1102, 2005.

[26] M. Polycarpou, J. Farrell, and M. Sharma, "On-line approximation control of uncertain nonlinear systems: issues with control input saturation," in Proceedings of the American Control Conference, pp. 543-548, June 2003.

[27] M. Chen, S. S. Ge, and B. Ren, "Adaptive tracking control of uncertain MIMO nonlinear systems with input constraints," Automatica, vol. 47, no. 3, pp. 452-465, 2011.

[28] Y.-L. Zhou and M. Chen, "Sliding mode control for NSVs with input constraint using neural network and disturbance observer," Mathematical Problems in Engineering, vol. 2013, Article ID 904830, 12 pages, 2013.

[29] Y. M. Li, T. S. Li, and S. C. Tong, "Adaptive fuzzy modular backstepping output feedback control of uncertain nonlinear systems in the presence of input saturation," International Journal of Machine Learning and Cybernetics, vol. 4, no. 5, pp. 527-536, 2013.

[30] Y. M. Li, S. C. Tong, and T. S. Li, "Direct adaptive fuzzy backstepping control of uncertain nonlinear systems in the presence of input saturation," Neural Computing and Applications, vol. 23, no. 5, pp. 1207-1216, 2013.

[31] Y. M. Li, S. C. Tong, and T. S. Li, "Adaptive fuzzy outputfeedback control for output constrained nonlinear systems in the presence of input saturation," Fuzzy Sets and Systems, vol. 248, pp. 138-155, 2014.

[32] D. Lin, X. Y. Wang, F. Z. Nian, and Y. L. Zhang, "Dynamic fuzzy neural networks modeling and adaptive backstepping tracking control of uncertain chaotic systems," Neurocomputing, vol. 73, no. 16-18, pp. 2873-2881, 2010.

[33] D. Lin, X. Wang, and Y. Yao, "Fuzzy neural adaptive tracking control of unknown chaotic systems with input saturation," Nonlinear Dynamics, vol. 67, no. 4, pp. 2889-2897, 2012.

[34] W. Z. Gao and R. R. Selmic, "Neural network control of a class of nonlinear systems with actuator saturation," IEEE Transactions on Neural Networks, vol. 17, no. 1, pp. 147-156, 2006. 
[35] R. Y. Yuan, J. Q. Yi, W. S. Yu, and G. L. Fan, "Adaptive controller design for uncertain nonlinear systems with input magnitude and rate limitations," in Proceedings of the American Control Conference (ACC '11), pp. 3536-3541, July 2011.

[36] R. Y. Yuan, X. M. Tan, G. L. Fan, and J. Q. Yi, "Robust adaptive neural network control for a class of uncertain nonlinear systems with actuator amplitude and rate saturations," Neurocomputing, vol. 125, pp. 72-80, 2014.

[37] B. Xiao, Q.-L. Hu, and G. Ma, "Adaptive sliding mode backstepping control for attitude tracking of flexible spacecraft under input saturation and singularity," Proceedings of the Institution of Mechanical Engineers, Part G: Journal of Aerospace Engineering, vol. 224, no. 2, pp. 199-214, 2010.

[38] C. Y. Wen, J. Zhou, Z. T. Liu, and H. Y. Su, "Robust adaptive control of uncertain nonlinear systems in the presence of input saturation and external disturbance," IEEE Transactions on Automatic Control, vol. 56, no. 7, pp. 1672-1678, 2011.

[39] S. Sui, S. C. Tong, and Y. M. Li, "Adaptive fuzzy backstepping output feedback tracking control of MIMO stochastic purefeedback nonlinear systems with input saturation," Fuzzy Sets and Systems, vol. 254, pp. 26-46, 2014.

[40] X. Wang, T. S. Li, L. Y. Fang, and B. Lin, "Adaptive NN control for a class of strict-feedback discrete-time nonlinear systems with input saturation," in Advances in Neural Networks-ISNN 2013, vol. 7952 of Lecture Notes in Computer Science, pp. 70-78, Springer, 2013.

[41] Z. Zhu, Y. Q. Xia, and M. Y. Fu, "Adaptive sliding mode control for attitude stabilization with actuator saturation," IEEE Transactions on Industrial Electronics, vol. 58, no. 10, pp. 48984907, 2011.

[42] Q. L. Hu, B. Xiao, and M. I. Friswell, "Robust fault-tolerant control for spacecraft attitude stabilisation subject to input saturation," IET Control Theory \& Applications, vol. 5, no. 2, pp. 271-282, 2011.

[43] G. Feng and R. Lozano, Adaptive Control Systems, Newnes, Oxford, UK, 1999.

[44] Y. N. Yang, J. Wu, and W. Zheng, "Attitude control for a station keeping airship using feedback linearization and fuzzy sliding mode control," International Journal of Innovative Computing, Information and Control, vol. 8, no. 12, pp. 8299-8310, 2012. 


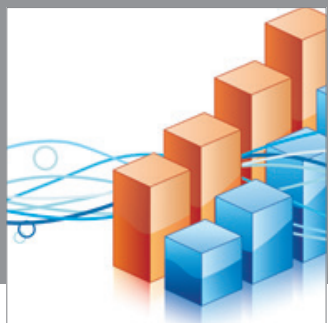

Advances in

Operations Research

mansans

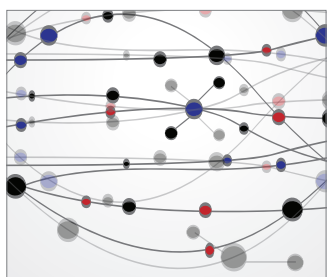

The Scientific World Journal
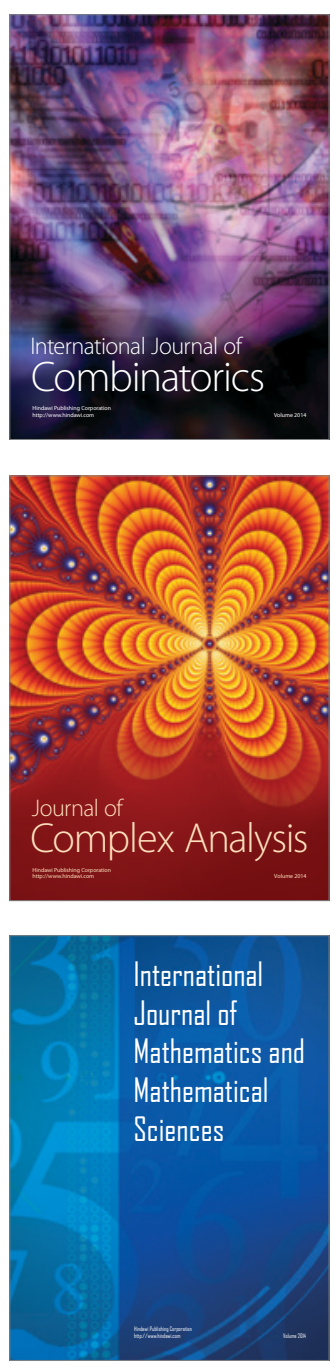
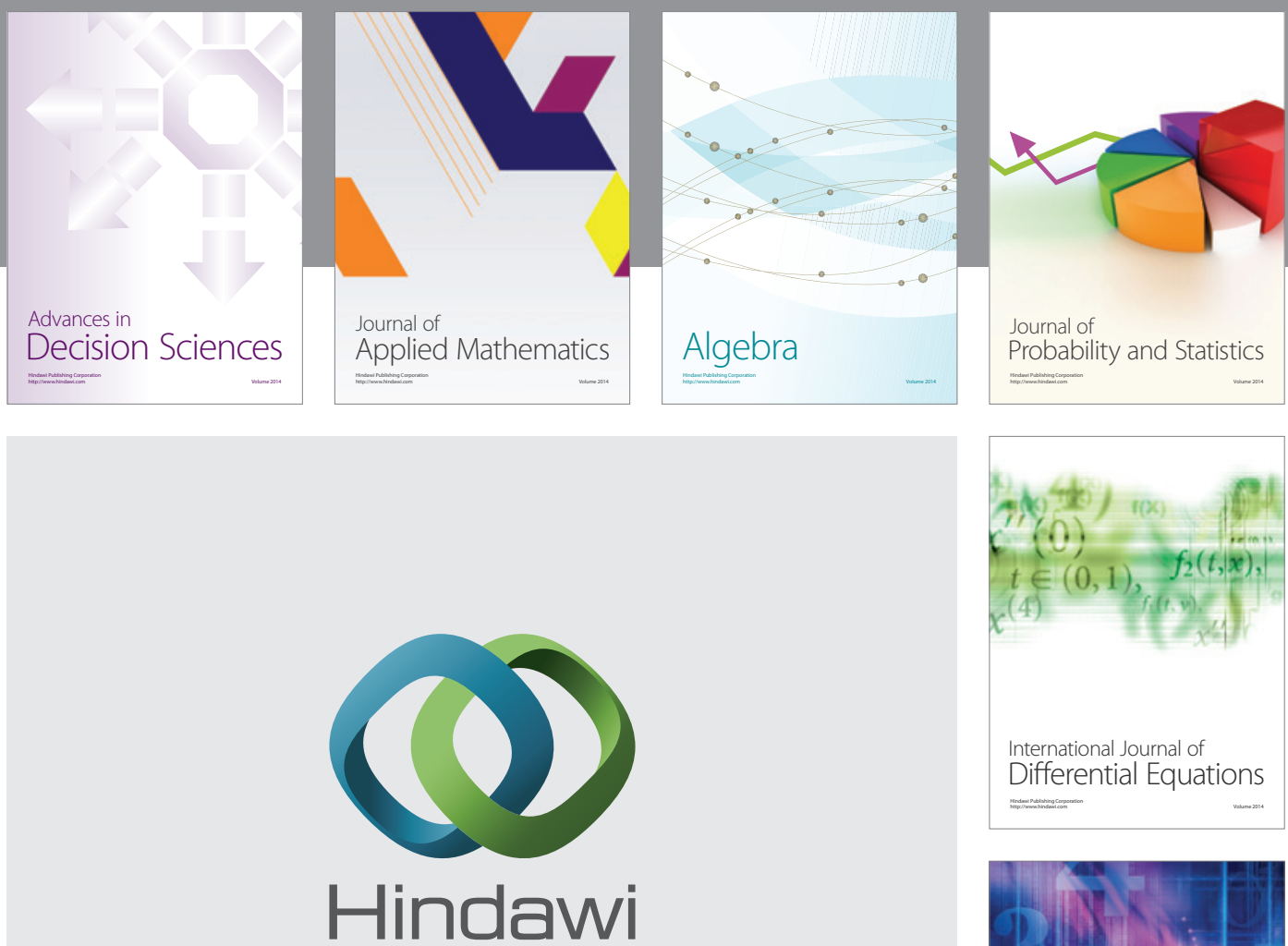

Submit your manuscripts at http://www.hindawi.com
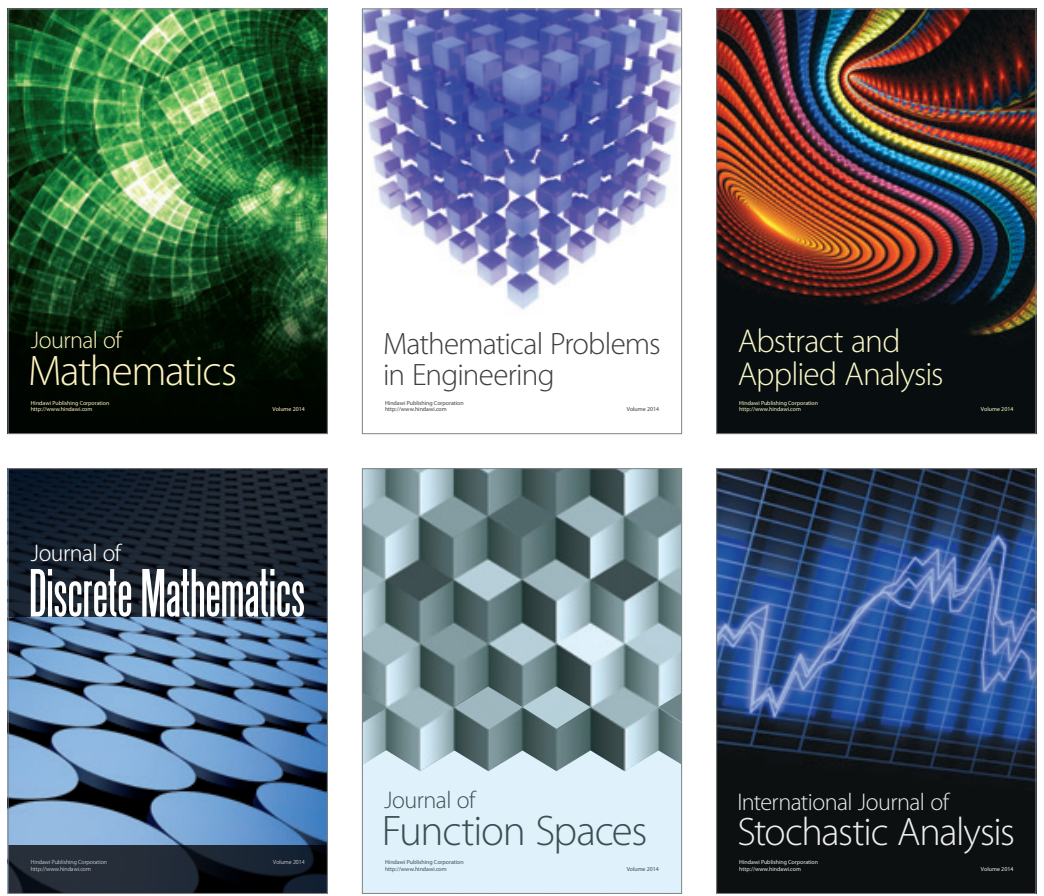

Journal of

Function Spaces

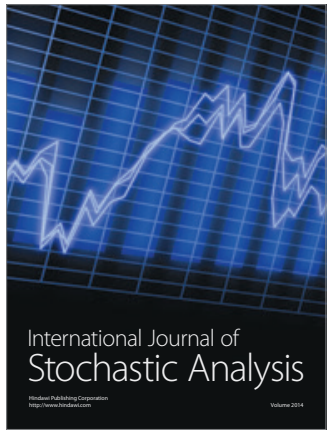

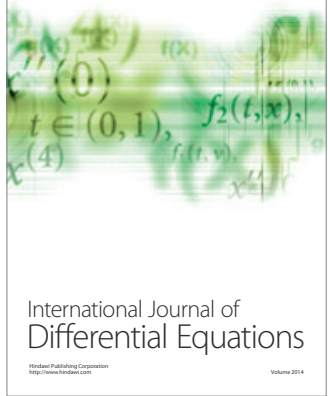
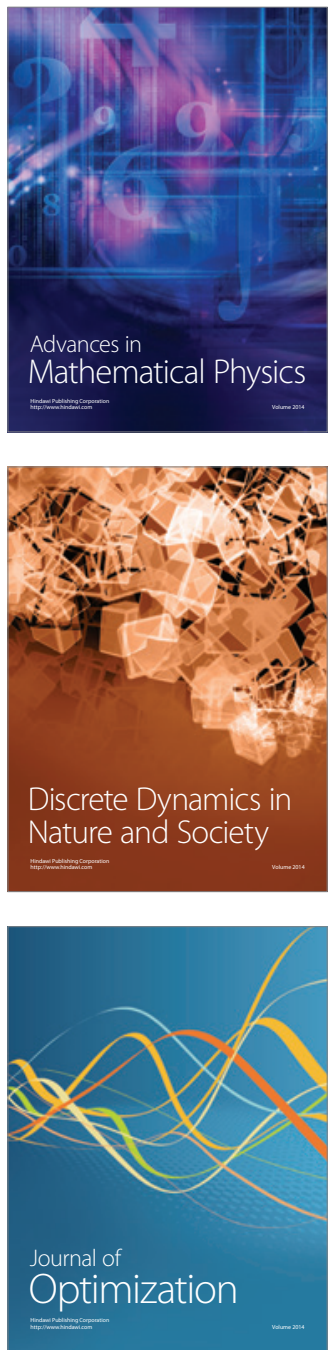